ISSN 1996-1944

www.mdpi.com/journal/materials

Review

\title{
Germanium Based Field-Effect Transistors: Challenges and Opportunities
}

\section{Patrick S. Goley * and Mantu K. Hudait}

Advanced Devices \& Sustainable Energy Laboratory (ADSEL), Bradley Department of Electrical and Computer Engineering, Virginia Tech, Blacksburg, VA 24061, USA; E-Mail: mantu.hudait@ vt.edu

* Author to whom correspondence should be addressed; E-Mail: patrick.goley@vt.edu; Tel.: +1-540-315-4767.

Received: 18 January 2014; in revised form: 6 March 2014 / Accepted: 7 March 2014 / Published: 19 March 2014

\begin{abstract}
The performance of strained silicon ( $\mathrm{Si}$ ) as the channel material for today's metal-oxide-semiconductor field-effect transistors may be reaching a plateau. New channel materials with high carrier mobility are being investigated as alternatives and have the potential to unlock an era of ultra-low-power and high-speed microelectronic devices. Chief among these new materials is germanium (Ge). This work reviews the two major remaining challenges that Ge based devices must overcome if they are to replace Si as the channel material, namely, heterogeneous integration of Ge on Si substrates, and developing a suitable gate stack. Next, Ge is compared to compound III-V materials in terms of $p$-channel device performance to review how it became the first choice for PMOS devices. Different Ge device architectures, including surface channel and quantum well configurations, are reviewed. Finally, state-of-the-art Ge device results and future prospects are also discussed.
\end{abstract}

Keywords: germanium; heterogeneous integration; passivation; buffer; high mobility; gate stack; quantum well

\section{Introduction}

The motivation to replace strained silicon $(\mathrm{Si})$ with higher mobility channel materials in today's metal-oxide-semiconductor field-effect transistors (MOSFETs) is well documented [1,2]. Saturation drive current $\left(I_{\mathrm{on}}\right)$, a critical performance metric for FET devices, is intimately linked with carrier mobility. While this link has become less clear as devices are scaled down into quasi-ballistic 
and ballistic regimes, the general relation continues to hold [3]. In light of this relationship, future transistor scaling will require the introduction of new high mobility channel materials, including germanium (Ge) or III-V semiconductors. The higher carrier mobility offered by these materials compared to strained silicon presents a practical means to push $I_{\text {on }}$ higher, even at lower supply voltages. On the other hand, even with the change in device architecture from planar to F in FET, continued scaling of today's Si transistors is expected to provide only a few additional generations of technology. As seen in Figure 1, the hole mobility enhancement as a function of strain is much flatter in Si than for Ge. This suggests that if the challenges associated with Ge channel transistors can be overcome, the technology may continue to be scalable for decades.

The effort to move to higher mobility channel materials is complicated by the fact that it must, in a sense, be done twice, once for $n$-channel FETs (NMOS) and once for $p$-channel FETs (PMOS). The nature of complementary MOS logic (CMOS) requires comparably performing NMOS and PMOS devices working in tandem. Historically, NMOS transistors have outperformed PMOS since electron mobility $\left(\mu_{\mathrm{e}}\right)$ is generally significantly higher than hole mobility $\left(\mu_{\mathrm{h}}\right)$. Circuit designers have adapted to working with PMOS devices with about one third the performance of NMOS, so preferably the performance gap in the next generation of technology should be no larger than this [1]. Additionally, it is strongly favorable to use the same material system for both $n$-channel and $p$-channel devices (either Ge for both, or for example, InGaAs for both) since this substantially simplifies device processing [4,5]. Due to the exceptionally high $\mu_{\mathrm{h}}$ of $\mathrm{Ge}$, and the progress made in Ge based $p$-channel MOSFETs (pMOSFETs) [6-14] and $p$-channel quantum well FETs (pQWFETs) [2,15-19] over the last decade, there appears to be a consensus in the device research community and in industry that $\mathrm{Ge}$ offers the best option for PMOS devices [1,2,20]. In light of this, there is heightened incentive to develop Ge based NMOS devices that perform comparably. There are, however, significant additional engineering challenges to achieving this, such as overcoming Ge's tendency for have a higher interface state density $\left(D_{\text {it }}\right)$ near the conduction band edge [21,22] and developing low resistance ohmic contacts to n-type Ge [23,24].

High mobility III-V materials present an alternative to Ge for future CMOS technology nodes. In III-V material systems, experimental outcomes have been the inverse of that seen with Ge-i.e., excellent III-V based NMOS devices have been demonstrated [25,26], whereas a comparably performing PMOS device using the same channel material is still elusive to date [1]. This is in large part due to the significant disparity in electron and hole mobility in III-V materials; with $\mu_{\mathrm{e}}$ typically being at least several times larger than $\mu_{\mathrm{h}}[1]$. For this reason, it is not unlikely that a future CMOS technology node will involve co-integration of Ge based PMOS and III-V based NMOS devices together. Unfortunately, such a path would also entail finding ways to deal with the increased processing complexity that comes with having multiple material systems with different chemistries and thermodynamic stability on the same chip. 
Figure 1. Hole mobility of $\mathrm{Si}$ and $\mathrm{Ge}$ as a function of stress and wafer orientation. Ge offers both better intrinsic hole mobility and better scalability compared to Si, reprinted with permission from [20]. Copyright 2012, IEEE.

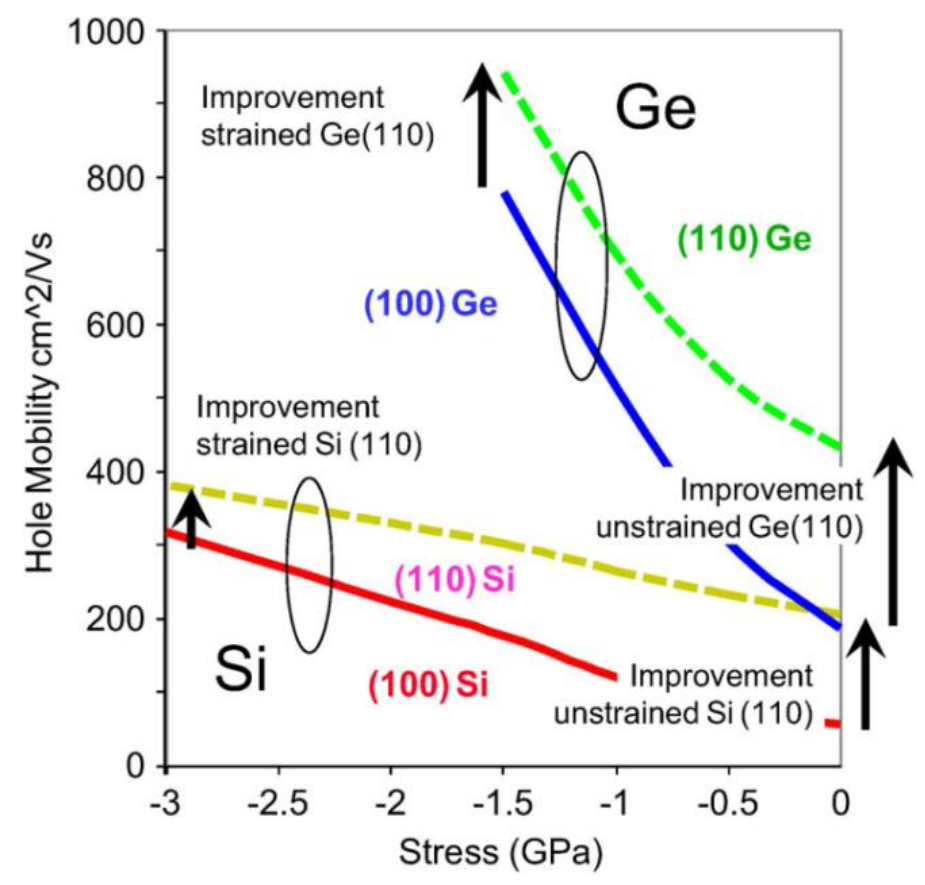

The above discussion has led to a choice of two paths, the dual-Ge path (for both NMOS and PMOS) or the III-V/Ge path (NMOS/PMOS). To be sure, there is also a possibility of a dual-III-V path; or it may be found the performance benefit of Ge and III-V over strained silicon (which is itself a moving target) fails to justify the increase in complexity and cost. The former seems the least likely of all alternatives, and the latter falls outside the scope of the present article. This review first summarizes the two major challenges that must be overcome to succeed in either path involving Ge: heterogeneous integration of $\mathrm{Ge}$ on $\mathrm{Si}$, and developing a suitable gate stack. After this, a major challenge to the dual-Ge path, achieving low resistance ohmic contacts on n-type Ge, is briefly discussed. The basis for Ge's selection over III-V for PMOS is also summarized. Finally, Ge device architectures and state-of-the-art results are reviewed. Many excellent review articles already exist for Ge based devices, with most focusing on the MOSFET architecture [2,11,27-30]. This review article presents recent advancements in the field of Ge based nanoscale MOSFETs and QWFETs.

\section{Challenges}

\subsection{Heterogeneous Integration on Silicon}

Due to low economic availability, any long-term widespread utilization of Ge for electronic device applications will almost certainly be in the form of thin epitaxial films grown on cheaper and more abundant $\mathrm{Si}$ substrates. Integration on $\mathrm{Si}$ is doubly important due to the expectation that other $\mathrm{Si}$ components, such DRAM and non-volatile memory, will be included on the same chips [31]. As a consequence, perhaps the most significant hurdle to the widespread adoption of Ge in devices is the lattice mismatch with respect to $\mathrm{Si}$. This problem is further aggravated by the thermal expansion coefficient mismatch in these two materials. Lattice mismatch is defined as: 


$$
\frac{\Delta a}{a}=\frac{a_{\mathrm{e}}-a_{\mathrm{s}}}{a_{\mathrm{s}}}=\frac{a_{\mathrm{Ge}}-a_{\mathrm{Si}}}{a_{\mathrm{Si}}}=\frac{5.658-5.431}{5.431}=4.18 \%
$$

where $a_{\mathrm{e}}$ is the lattice parameter of the epitaxial layer and $a_{\mathrm{s}}$ is that of the substrate material. The lattice mismatch leads to a large buildup of strain energy in Ge layers epitaxially grown on Si. This strain energy is primarily relieved by two mechanisms: (i) generation of lattice dislocations at the interface (misfit dislocations) and (ii) elastic deformation of both the substrate and the Ge islands which form on the surface during early stages of growth (following the Stranski-Krastanow growth mode) [32]. In general, elastic deformation cannot accommodate all the strain and as a result, misfit dislocations nucleate at the island edges [33]. Many dislocations bend toward the growth direction and propagate, or thread, to the surface during growth. These threading dislocations severely degrade carrier transport properties by lowering carrier mobility and decreasing carrier lifetime. This dilemma necessitates the development of means to more gracefully bridge the lattice constants of the two materials. To accomplish this, several buffer architectures have been proposed and experimentally demonstrated. These approaches are summarized in Table 1. While not complete, Table 1 does highlight the wide variety of methods that have been explored for Ge on Si heteroepitaxy. Thinner buffers are generally preferred to reduce cost and facilitate heat dissipation. For FET applications, the buffer should be comprised of wide bandgap materials to (i) provide device isolation and (ii) suppress parallel conduction - two phenomena discussed in more detail later. A good buffer is further characterized by a low threading dislocation density (TDD) and low root-mean-square (RMS) roughness at the surface so that it may serve as a smooth template for active layer growth. It is believed that for device quality growth, a starting surface with a TDD of $10^{6} \mathrm{~cm}^{-2}$ or less, and a RMS roughness of $1 \mathrm{~nm}$ or less, is required. The approaches summarized in Table 1 generally fall into five categories: (i) direct epitaxy of $\mathrm{Ge}$ on $\mathrm{Si}$, (ii) growing layers of $\mathrm{Si}_{1-x} \mathrm{Ge}_{x}$ where $x$ is graded from zero to one, (iii) surfactant mediated epitaxy (SME), (iv) aspect ratio trapping (ART), and (v) the oxide buffer. Each of these architectures is briefly reviewed in the following sections. It should be pointed out that buffer architectures demonstrating heterogeneous integration of GaAs on $\mathrm{Si}$ may also prove useful for Ge. This is due to the close lattice match between GaAs and Ge $(0.07 \%)$, which significantly reduces the complexity of $\mathrm{Ge}$ on GaAs growth [34]. An excellent review of GaAs on Si epitaxy can be found in [35]. The higher bandgap of GaAs $(1.42 \mathrm{eV})$ compared to $\mathrm{Ge}(0.67 \mathrm{eV})$ suggests that GaAs-based buffers should exhibit less parallel conduction than Ge-based ones. One exciting GaAs/Si work been added to Table 1 [25].

Table 1. Summary of buffer architectures for Ge epitaxy on Si-based substrates.

\begin{tabular}{|c|c|c|c|c|c|c|}
\hline Category & Approach & Details & Substrate & $\begin{array}{c}\text { Bandgap of } \\
\text { buffer } \\
\text { material (eV) }\end{array}$ & $\begin{array}{l}\text { Total } \\
\text { thickness } \\
\text { (nm) }\end{array}$ & Ref. \\
\hline \multirow[b]{2}{*}{$\begin{array}{l}\text { Direct } \\
\text { Epitaxy }\end{array}$} & $\begin{array}{l}\text { Multiple hydrogen } \\
\text { annealing for } \\
\text { heteroepitaxy (MHAH) }\end{array}$ & $\begin{array}{l}\text { direct epitaxy of } \mathrm{Ge} \text {; multiple cycles of } \\
\text { growth }\left(400^{\circ} \mathrm{C}\right) \text { and high temperature } \\
\text { hydrogen annealing }\left(700-800^{\circ} \mathrm{C}\right)\end{array}$ & $\begin{array}{l}\text { standard bulk } \\
\mathrm{Si}\end{array}$ & 0.67 & 400 & {$[36,37]$} \\
\hline & $\begin{array}{l}\text { Multiple cycles of low } \\
\text { temp, high temp, } \\
\text { hydrogen annealing }\end{array}$ & $\begin{array}{l}\text { (i) deposit Ge directly on Si at low temp; } \\
\text { (ii) deposit Ge at high temp onto low temp } \\
\text { layer; (iii) high temperature hydrogen } \\
\text { annealing; (iv) repeat (i) to (iii) } 3 \text { or } 4 \text { times }\end{array}$ & $\begin{array}{l}\mathrm{Si}(100) 4^{\circ} \\
\text { offcut towards } \\
<110>\end{array}$ & 0.67 & $\begin{array}{l}2400 \\
(4 \text { cycles }) \\
1400 \\
(3 \text { cycles })\end{array}$ & [38] \\
\hline
\end{tabular}


Table 1. Cont.

\begin{tabular}{|c|c|c|c|c|c|c|}
\hline Category & Approach & Details & Substrate & $\begin{array}{c}\text { Bandgap of } \\
\text { buffer } \\
\text { material (eV) } \\
\end{array}$ & $\begin{array}{c}\text { Total } \\
\text { thickness } \\
\text { (nm) }\end{array}$ & Ref. \\
\hline \multirow{3}{*}{$\begin{array}{l}\text { Graded } \\
\text { SiGe } \\
\text { Buffer }\end{array}$} & $\begin{array}{l}\text { Step graded } \mathrm{Si}_{1-x} \mathrm{Ge}_{x} \\
\text { buffer }\end{array}$ & $\begin{array}{l}0.8 \mathrm{um} \mathrm{Si}_{0.1} \mathrm{Ge}_{0.9} \text {, followed by } 0.8 \mathrm{um} \\
\mathrm{Si}_{0.05} \mathrm{Ge}_{0.95} \text {, followed by } 1 \mathrm{um} \mathrm{Ge} \text { (all at } \\
\left.400{ }^{\circ} \mathrm{C}\right) ; 750{ }^{\circ} \mathrm{C} \text { annealing between layers. }\end{array}$ & $\begin{array}{l}\text { Si }(100) 6^{\circ} \\
\text { offcut towards } \\
<110>\end{array}$ & $0.67-1.12$ & 3600 & [39] \\
\hline & $\begin{array}{l}\text { Step graded } \mathrm{Si}_{1-x} \mathrm{Ge}_{x} \\
\text { buffer }\end{array}$ & $\begin{array}{l}\text { (i) } 0.5-1.0 \mathrm{um} \mathrm{Si}_{0.7} \mathrm{Ge}_{0.3} \text { layer grown on } \mathrm{Si} \text {; } \\
\text { (ii) } 0.3 \text { to } 1.0 \mathrm{um} \mathrm{Si}_{0.3} \mathrm{Ge}_{0.7} \text {; (iii) } 10-50 \mathrm{~nm} \\
\text { phos. doped } \mathrm{Si}_{0.3} \mathrm{Ge}_{0.7} \text { to suppress parallel } \\
\text { conduction; (iv) } 0.5-3.0 \mathrm{um} \mathrm{Si}_{0.3} \mathrm{Ge}_{0.7} \text {. }\end{array}$ & $\mathrm{Si}$ & $0.67-1.12$ & $\begin{array}{l}\sim 1550 \text { (est. } \\
\text { from TEM } \\
\text { image) }\end{array}$ & [15] \\
\hline & $\begin{array}{l}\text { Continuously Graded } \\
\mathrm{Si}_{1-x} \mathrm{Ge}_{x} \text { and chemical- } \\
\text { mechanical polishing } \\
(\mathrm{CMP})\end{array}$ & $\begin{array}{l}\text { (i) Graded from } \mathrm{Si} \text { to } \mathrm{Si}_{0.5} \mathrm{Ge}_{0.5} \text { at } 10 \% \mathrm{Ge} \\
\mathrm{um}^{-1} \text {; (ii) } 1.5 \mu \mathrm{m} \mathrm{Si} i_{0.5} \mathrm{Ge}_{0.5} \text { cap; (iii) top } 5 \mu \mathrm{m} \\
\text { removed by CMP; (iv) } \mathrm{Si}_{0.5} \mathrm{Ge}_{0.5} \text { graded to } \\
\mathrm{Si}_{0.08} \mathrm{Ge}_{0.92} \text { at } 10 \% \mathrm{Ge} \mathrm{um}{ }^{-1} \text {; (v) } 1.5 \mathrm{um} \mathrm{Ge} \\
\text { cap. }\end{array}$ & $\begin{array}{l}\operatorname{Si}(100) 6^{\circ} \\
\text { offcut towards } \\
<110>\end{array}$ & $0.67-1.12$ & 12000 & [40] \\
\hline $\begin{array}{l}\text { Surfactant } \\
\text {-Mediated } \\
\text { Epitaxy }\end{array}$ & $\begin{array}{l}\text { A surfactant }(\mathrm{Sb}) \text { is used } \\
\text { to alter the strain-relief } \\
\text { mechanism in Ge on } \mathrm{Si} \\
\text { epitaxy }\end{array}$ & $\begin{array}{l}\text { One monolayer (ML) of } \mathrm{Sb} \text { is deposited on } \\
\text { the } \mathrm{Si} \text { substrate followed by } 1 \mu \mathrm{m} \text { of } \mathrm{Ge} \text {; a } \\
\text { high } \mathrm{Sb} \text { flux of } \sim 2.4 \mathrm{ML} / \mathrm{min} \text { is maintained } \\
\text { during Ge growth. }\end{array}$ & $\operatorname{Si}(100)$ & 0.67 & $\sim 1000$ & [41] \\
\hline $\begin{array}{l}\text { Aspect } \\
\text { Ratio } \\
\text { Trapping }\end{array}$ & $\begin{array}{l}\text { ART combined with } \\
\text { epitaxial lateral } \\
\text { overgrowth (ELO) and } \\
\text { CMP }\end{array}$ & $\begin{array}{l}\text { Ge is grown in patterned trenches in } \\
\text { thermally grown } \mathrm{SiO}_{2} \text {; dislocations terminate } \\
\text { on trench sidewalls; lateral Ge growth begins } \\
\text { beyond trench height; CMP used to flatten } \\
\text { the surface. }\end{array}$ & $\operatorname{Si}(100)$ & 0.67 & $\sim 1000$ & [42] \\
\hline \multirow[b]{2}{*}{$\begin{array}{l}\text { Oxide } \\
\text { Buffer }\end{array}$} & $\begin{array}{l}\text { Ge condensation } \\
\text { technique }\end{array}$ & $\begin{array}{l}\text { Epitaxial SiGe layers are grown on } \\
\text { silicon-on-insulator (SOI) substrates } \\
\text { followed by successive thermal oxidation. }\end{array}$ & $\begin{array}{l}\text { strained and } \\
\text { unstrained } \\
\text { SOI }\end{array}$ & not reported & $\begin{array}{l}\text { not } \\
\text { reported }\end{array}$ & [43] \\
\hline & Epitaxial SHTO buffer & $\begin{array}{l}\text { (i) Epitaxial } \mathrm{SrTiO}_{3} \text { is deposited using } \\
\text { method in ref. [44]; (ii) epitaxial } \\
\mathrm{SrHf}_{x} \mathrm{Ti}_{x-1} \mathrm{O}_{3} \text { grown; (iii) Ge grown in two } \\
\text { steps, } 610{ }^{\circ} \mathrm{C} \text { for nucleation, then } 350{ }^{\circ} \mathrm{C} \text { for } \\
\text { homogenous coverage. }\end{array}$ & $\operatorname{Si}(100)$ & $\begin{array}{l}3.5(\mathrm{STO}) \\
6.5(\mathrm{SHO}) \\
{[45]}\end{array}$ & $\begin{array}{l}\sim 20 \text { (est. } \\
\text { from TEM } \\
\text { image) }\end{array}$ & [46] \\
\hline $\begin{array}{l}\text { GaAs } \\
\text { Buffer }\end{array}$ & $\begin{array}{l}\text { GaAs nucleation and } \\
\text { buffer layer }\end{array}$ & $\begin{array}{l}\text { An InGaAs QWFET heterogeneously } \\
\text { integrated on Si was demonstrated using this } \\
\text { buffer. }\end{array}$ & $\begin{array}{l}\mathrm{Si}(100) 4^{\circ} \\
\text { offcut towards } \\
<110>\end{array}$ & 1.42 & 500 & [25] \\
\hline
\end{tabular}

\subsubsection{Direct Epitaxy}

The direct epitaxy approach is attractive due to its relative simplicity, and with the aid of high temperature hydrogen annealing, this method has demonstrated films with low RMS surface roughness (less than $1 \mathrm{~nm}$ ) [38]. In regards to defect density however, direct epitaxy methods have had mixed results [47]. This method often involves a two-step low temperature then high temperature (LT/HT) Ge growth process. First a low temperature, low growth rate "seed" layer is grown which is intended to confine most of the defects. Together with Ge's low surface diffusivity, lowering the growth temperature tends to prevent the impinging Ge from forming islands on the surface, resulting in flatter 
and more homogenous coverage of the Si substrate. After approximately $30 \mathrm{~nm}$ of growth the strain in the Ge surface will be fully relaxed due to the generation of misfit dislocations [48]. After the low temperature layer, a high temperature and high growth rate layer is deposited and is expected to be of higher crystalline quality since (i) the higher growth temperature annihilates defects and dislocations associated with strain relaxation, and (ii) the Ge is growing on a fully relaxed Ge "seed" layer instead of $\mathrm{Si}$.

Separately from the two-step LT/HT approach, Nayfeh et al. [37] clearly demonstrated the benefit of hydrogen annealing of Ge layers on $\mathrm{Si}$. The group reported an $88 \%$ reduction in RMS roughness for a $200 \mathrm{~nm}$ thick Ge layer grown on $\mathrm{Si}$ (at $400{ }^{\circ} \mathrm{C}$ ) that was annealed for 1 hour at $825{ }^{\circ} \mathrm{C}$ in $\mathrm{H}_{2}$, compared to no reduction for a sample annealed in $\mathrm{N}_{2}$. They attributed the improved flatness to the increased surface mobility of the Ge-H cluster compared to Ge alone. Interestingly, the group found the ratio of optimal annealing temperatures for $\mathrm{Si}$ and $\mathrm{Ge}(1423 \mathrm{~K} / 1073 \mathrm{~K}=1.326)$ to be nearly equal to the ratio of their melting points $(1687 \mathrm{~K} / 1211 \mathrm{~K}=1.393)$. In a separate paper [36] the same group used this approach to fabricate a Ge pMOSFET. They have grown a second Ge layer $\left(400{ }^{\circ} \mathrm{C}, 250 \mathrm{~nm}\right.$ thick) on top of a Ge layer produced by the method described in their previous work [37]. The resulting structure is shown in Figure 2. They observed a $2 \times$ increase in effective low-field mobility and a $3 \times$ increase in effective high-field mobility in a fabricated Ge pMOSFET compared to $\mathrm{Si}$. Recently, this $\mathrm{Ge}$ on $\mathrm{Si}$ growth method was used in combination with a patterned $\mathrm{SiO}_{2}$ mask to achieve selective-area growth of Ge on $\mathrm{Si}$ and obtained a TDD of $1 \times 10^{7} \mathrm{~cm}^{-2}$ and RMS roughness of $0.61 \mathrm{~nm}$ [49]. Hartmann et al. [50] reported a Ge surface with a TDD of $6 \times 10^{6} \mathrm{~cm}^{-2}$ and RMS roughness of $\sim 1 \mathrm{~nm}$. The group used a similar two-step LT/HT growth process $\left(400{ }^{\circ} \mathrm{C} / 750{ }^{\circ} \mathrm{C}\right)$ and the total buffer thickness was $2.5 \mu \mathrm{m}$. Following growth, the structure was cyclically annealed (eight times $750{ }^{\circ} \mathrm{C}-10 \mathrm{~min} / 900{ }^{\circ} \mathrm{C}-10 \mathrm{~min}$ ) in $\mathrm{H}_{2}$. The hydrogen annealing was found to be critical for minimizing TDD, lowering it by nearly one order of magnitude (from $\sim 4 \times 10^{7}$ to $6 \times 10^{6} \mathrm{~cm}^{-2}$ ), but at the cost of a small increase in RMS roughness (increasing it from $\sim 0.7$ to $\sim 1.1 \mathrm{~nm}$ ). It should be noted that the group has assumed TDD to be equal to pit density measured by atomic force microscopy (AFM). Kobayashi et al. [51] observed more than one order of magnitude difference between the TDD measured by plan-view transmission electron microscopy (TEM) and the pit density measured by AFM. Choi et al. [38] have grown multiple two-step LT/HT dual layer stacks and have used $30 \mathrm{~min}$ hydrogen annealing at $800{ }^{\circ} \mathrm{C}$ between each set. They found the AFM pit density reduced by $80 \%$ between the first and second sets of LT/HT layers. After three sets of growth and $\mathrm{H}_{2}$ annealing (and a total Ge thickness of $\sim 1.81 \mu \mathrm{m}$ ) they achieved a TDD measured by plan-view TEM of $0.8-1 \times 10^{7} \mathrm{~cm}^{-2}$ and RMS roughness of $0.4-0.6 \mathrm{~nm}$. This is encouraging since additional sets of LT/HT growth and annealing could result in even lower TDD and RMS roughness, albeit with decreasing gains and additional buffer thickness.

Having a buffer composed of high bandgap material helps ensure that performance characteristics of a given device in an array are as much as possible independent of the on/off states of adjacent devices, or that is it electrically isolated from them. A pure Ge based buffer directly on Si would be expected to have poor device isolation characteristics due to the low bandgap of Ge. This alone is not necessarily mark against the direct epitaxy approach since it could be combined with commercially available silicon-on-insulator (SOI) substrates to improve device isolation, although this would also require that the buffer be quite thin to facilitate etching down to the buried oxide. A more serious 
concern for this approach is the prospect of parallel conduction of current through the buffer between the source and drain. An excellent review on the nature of parallel conduction can be found in [52]. The existence of additional higher conductivity paths between the source and drain, such as that provided by low bandgap buffer layers, has two primary consequences. First, it provides the means for a higher off-state current in FET devices, which in turn reduces the $I_{\mathrm{on}} / I_{\text {off }}$ ratio and contributes power losses. This is exacerbated by the fact that the conductivity of these secondary paths is not effectively modulated by the gate voltage. Second, it undermines the device's ability to confine carriers to the layer or path of highest mobility, which is particularly important in QWFET applications (discussed in section three). Failure to confine carriers to the highest mobility layer decreases on-state current, which also further reduces the device $I_{\mathrm{on}} / I_{\text {off }}$ ratio. More investigation is needed to determine if parallel conduction is serious issue for the direct epitaxy approach.

Figure 2. Cross-sectional TEM images of heteroepitaxial-Ge layers on $\mathrm{Si}$; (a) $155 \mathrm{~nm}$ of Ge grown in single growth cycle with one $\mathrm{H}$ anneal step and (b) $400 \mathrm{~nm}$ of Ge layer grown in two growth cycles with two $\mathrm{H}$ annealing cycles (MHAH method), reprinted with permission from [36]. Copyright 2005, IEEE.

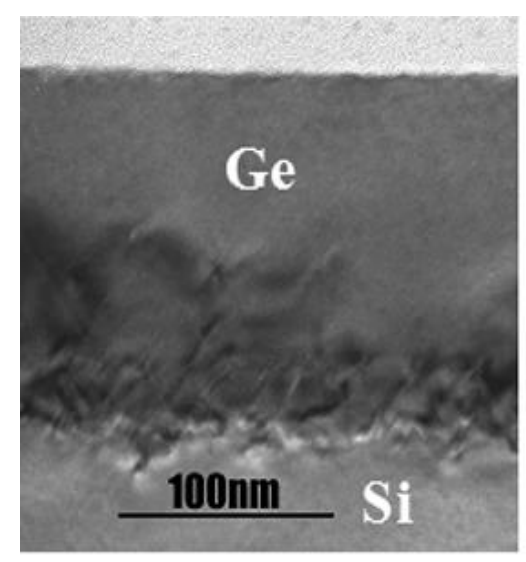

(a)

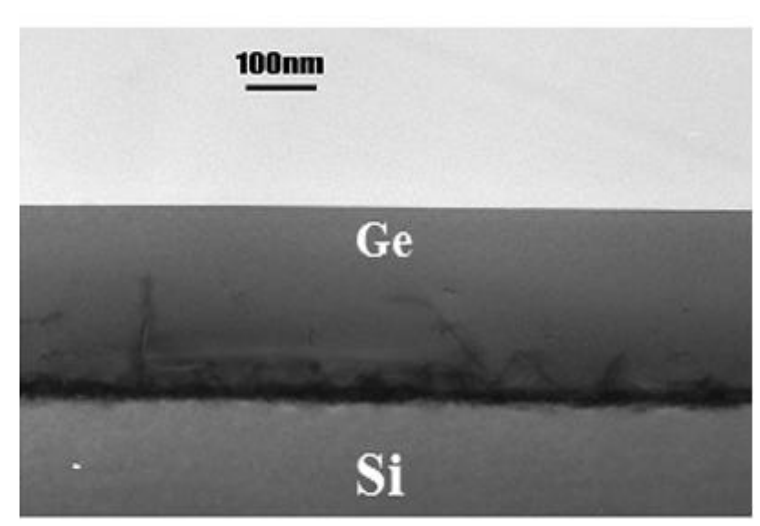

(b)

\subsubsection{The Graded $\mathrm{Si}_{1-x} \mathrm{Ge}_{x}$ Buffer}

The graded $\mathrm{Si}_{x} \mathrm{Ge}_{1-x}$ buffer approach aims to introduce the $4.2 \%$ lattice mismatch gradually rather than abruptly as in the direct epitaxy approach. This is possible since the lattice mismatch of $\mathrm{Si}_{1-x} \mathrm{Ge}_{x}$ on $\mathrm{Si}$ is roughly proportional to the Ge composition $(x)$. Furthermore, the threading dislocations created during growth of the initial low-Ge composition layers can be preserved and reused to relieve additional strain generated in subsequent higher-Ge composition layers by carefully controlling the grading rate and the growth temperature. The gliding of existing threading dislocations suppresses nucleation of additional dislocations. A notable implementation of this approach was carried out by Currie et al. [40]. The group achieved a remarkable TDD of $2.1 \times 10^{6} \mathrm{~cm}^{-2}$. However, the total buffer thickness was $12 \mu \mathrm{m}$ and the RMS roughness was $24.2 \mathrm{~nm}$. Additionally, the group introduced a chemical-mechanical-polish (CMP) step near the growth midpoint since it was observed that the dislocations had a tendency to pile-up and become trapped, and therefore unable to continue to facilitate strain relief in subsequent layers. Samples with this added CMP step showed lower TDD of $\sim 2.1 \times 10^{6} \mathrm{~cm}^{-2}$ and lower RMS roughness of $24.2 \mathrm{~nm}$ (compared to $1-5 \times 10^{7} \mathrm{~cm}^{-2}$ and $47 \mathrm{~nm}$ 
without the CMP). Loh et al. [53] later combined the graded SiGe buffer approach and the LT/HT approach to obtain an estimated TDD of $6 \times 10^{6} \mathrm{~cm}^{-2}$ (estimated by etch pit density) and RMS roughness of $1.4 \mathrm{~nm}$ using a buffer of only about $160 \mathrm{~nm}$.

To help address the concern of device isolation, the graded SiGe buffer approach could be carried out on silicon on insulator (SOI) substrates, as shown by the Loh group [53]. As with the direct epitaxy approach, more experiments are need to investigate to nuisance of parallel conduction.

\subsubsection{Surfactant Mediated Epitaxy (SME)}

The amount of strain in epitaxial layers strongly influences the growth mode of the film. In the case of $\mathrm{Ge}$ on $\mathrm{Si}$, the growth is flat (2D) for the first few monolayers. As the built-in strain energy increases with increasing film thickness, there is a sudden transition from 2D to 3D growth modes, also known as island growth [33]. It has been shown that this 2D-to-3D growth mode transition can be suppressed by deliberate introduction of a surfactant to alter the surface free energy, resulting in smoother layer-by-layer growth [54].

The general procedure for SME is as follows. The Si substrate is first capped with $\sim 1$ ML of the surfactant. The first surfactant investigated for Ge/Si growth was As. The extra valence electron from the As in the capping layer fills the dangling bonds at the $\mathrm{Si}$ surface, resulting in a stable, or energetically favorable termination. Following capping layer deposition, Ge growth begins. Surface termination with As remains energetically favorable since the Ge surface also contains dangling bonds. As a result, Ge atoms on the surface efficiently exchange sites with As in the capping layer. By this process the As capping layer "floats" on the surface even as Ge atoms continue to impinge on the surface, and the surface mobility of the growing species is strongly diminished, effectively suppressing the ability of the Ge to form islands. A drawback of the SME approach is that some of the surfactant inevitably incorporates into the film bulk, leading to a moderate $\left(10^{16}-10^{19} \mathrm{~cm}^{-3}\right) \mathrm{n}$-type background doping.

In thicker films where islanding occurs, many defects thread towards the surface where the islands eventually coalesce. Using a surfactant to preserve the 2D growth mode also alters the defect structure that forms to relieve the lattice strain. LeGoues et al. [33] found that when As is used as the surfactant during $\mathrm{Ge}$ on $\mathrm{Si}(100)$ growth it results in $\mathrm{V}$-shaped defects that can generate stacking faults and twins that extend throughout the entire film. Horn-von Hoegen et al. [55] showed that when Sb is used as the surfactant for $\mathrm{Ge} / \mathrm{Si}(111)$ heteroepitaxy the threading defects generated to relieve stain during initial stages later self-annihilate as growth proceeds. This results in a network of dislocations confined at the $\mathrm{Ge} / \mathrm{Si}(111)$ interface, and comparatively much fewer defects in the bulk of the film. A TDD of less than $10^{8} \mathrm{~cm}^{-2}$ and background doping of $3-4 \times 10^{19} \mathrm{~cm}^{-3}$ were estimated. More recently, Wietler et al. [41] used $\mathrm{Sb}$ for $\mathrm{Ge}$ on $\mathrm{Si}(100)$ growth to achieve fully relaxed Ge layers with a TDD of $1.6 \times 10^{8} \mathrm{~cm}^{-2}$ measured by plain-view TEM. The group used a relatively high substrate growth temperature of $670{ }^{\circ} \mathrm{C}$ and $\mathrm{Sb}$ flux of $2.4 \mathrm{ML} / \mathrm{min}$. The high temperature helped facilitate $\mathrm{Sb}$ segregation to the surface, leading to a relatively low background doping of $3-4 \times 10^{16} \mathrm{~cm}^{-3}$ in the Ge film. To further address the issue of surfactant incorporation in the Ge layer, it has recently been proposed to use carbon as the surfactant in place of Sb since carbon in Ge (i) does not act as a dopant and (ii) has very low solid solubility [56,57]. 


\subsubsection{Aspect Ratio Trapping (ART)}

The ART approach, also called "epitaxial necking" [58], for Ge-on-Si heteroepitaxy is built on the premise that most threading dislocations do not run normal to the Ge/Si interface. Therefore, by growing $\mathrm{Ge}$ on $\mathrm{Si}$ within narrow trenches formed by patterned oxide, dislocations angled away from the normal are likely to eventually collide with and become "trapped" by the trench sidewall.

ART begins by thermally growing $0.5-1 \mu \mathrm{m}$ of oxide on the substrate. Trenches are formed in the oxide using standard lithography and reactive ion etching (RIE). Bai et al. [59] developed a model to determine the optimal dimensions, or aspect ratio ( $A R=$ height/width), of the trenches. Park et al. [60] showed that trenches with $A R>1$ were needed to effectively trap defects within the sidewalls. A drawback of ART growth is that, while the length of trenches is arbitrary, the width of the area available for Ge devices is restricted to less than $1 \mu \mathrm{m}$ by the $A R>1$ requirement and the need for relatively thin buffers. To obtain a larger area of high-quality Ge for device growth, the Ge can be overgrown beyond the trench height, resulting in epitaxial lateral overgrowth (ELO). However, when ELO emerging from adjacent trenches coalesces, it forms new defects, as shown in Figure 3c(3). Defects originating at the Ge/Si interface in the trench (1) do not appear to propagate beyond the trench height (2). To avoid the defects formed by ELO coalescence, Park et al. [42] later used a growth process optimized for lateral growth, and CMP, to develop long $20 \mu \mathrm{m}$ wide regions of Ge, grown from a single trench, with a TDD of $1.6 \times 10^{6} \mathrm{~cm}^{-3}$, shown in Figure 3a,b. For some device applications long $20 \mu \mathrm{m}$ wide patches of high quality Ge may be sufficient [61]. If device area continues to improve, ART may become a viable method for creating Ge/Si virtual substrates for future high-performance Ge CMOS devices.

Figure 3. XTEM micrographs of Ge/Si heteroepitaxy using the ART method. (a,b) Uncoalesced Ge grown by epitaxial lateral overgrowth (ELO) from a single trench (indicated by a black arrow). The surface has been flattened using CMP; (c) ART Ge/Si in which growth from adjacent overgrown trenches has coalesced, reprinted with permission from [42]. Copyright 2009, The Electrochemical Society.
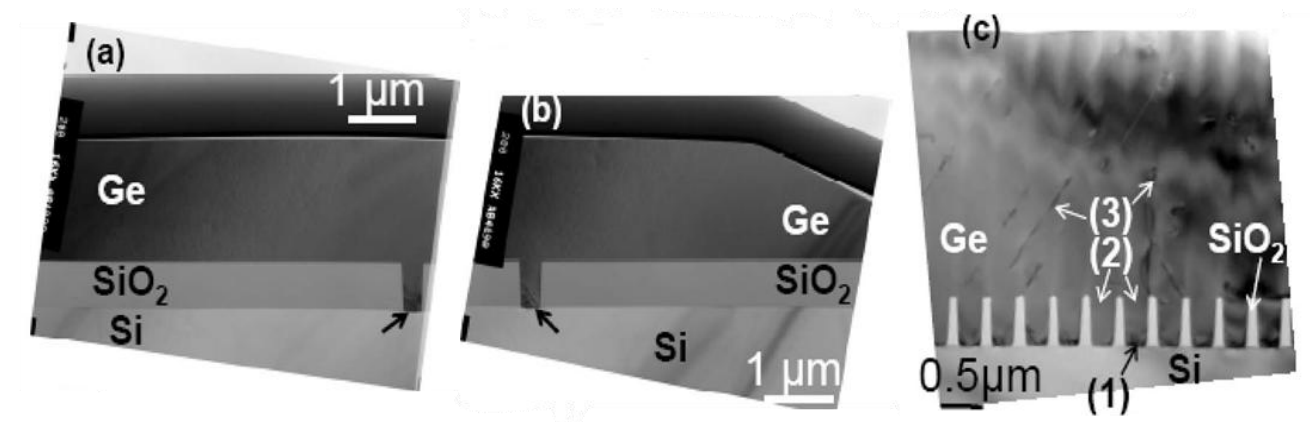

\subsubsection{The Oxide Buffer}

Two distinctly different oxide-based approaches have emerged for heterogeneous integration of Ge on Si: (i) the epitaxial oxide buffer [46,62-64], and (ii) heteroepitaxial growth of Ge on high Ge content SiGe-on-insulator (SGOI) substrates [43,65-67]. 
The epitaxial oxide based buffers are especially appealing due to (i) device isolation and (ii) the natural suppression of parallel conduction being inherently built-in to the structure. However, there is limited literature available on this method and more experiments are needed to clearly demonstrate its merit. Bojarczuk et al. [64] obtained a single-crystalline fully-relaxed layer (4 $\mathrm{nm})$ of Ge on a $\left(\mathrm{La}_{x} \mathrm{Y}_{1-x}\right)_{2} \mathrm{O}_{3} / \mathrm{Si}(111)$ template. Amorphous $\mathrm{Ge}$ was deposited on the crystalline $\left(\mathrm{La}_{x} \mathrm{Y}_{1-x}\right)_{2} \mathrm{O}_{3}$ and subsequently heated to $450{ }^{\circ} \mathrm{C}$ under an $\mathrm{Sb}$ flux to induce an amorphous to crystalline transformation of the Ge. The Sb flux was found to reduce surface roughening during recrystallization. The quality of the Ge surface, in terms of RMS roughness and TDD, was not reported. Seo et al. [46] used a two stage $\operatorname{SrHf}_{x} \mathrm{Ti}_{1-x} \mathrm{O}_{3}$ buffer on $\mathrm{Si}(100)$ to obtain a continuous and flat epitaxial Ge film, shown in Figure 4. The group conveyed that the method needed improvement due to low Hall-mobility $\left(100-300 \mathrm{~cm}^{2} / \mathrm{Vs}\right)$ and a high density of stacking faults and twin structures $\left(10^{9} \mathrm{~cm}^{-2}\right)$. Giussani et al. [63] used $\mathrm{PrO}_{2} / \mathrm{Si}(111)$ as a template for $\mathrm{Ge}(111)$ epitaxy and obtained a smooth single-crystalline Ge layer. However, an extended Ge deposition time was needed. Quantitative measurements of the surface quality were not provided.

Figure 4. HRTEM image of Ge grown on SHO. The interface between Ge and SHO is atomically sharp and contains no interfacial layer (inset), reprinted with permission from [46]. Copyright 2007, Elsevier.

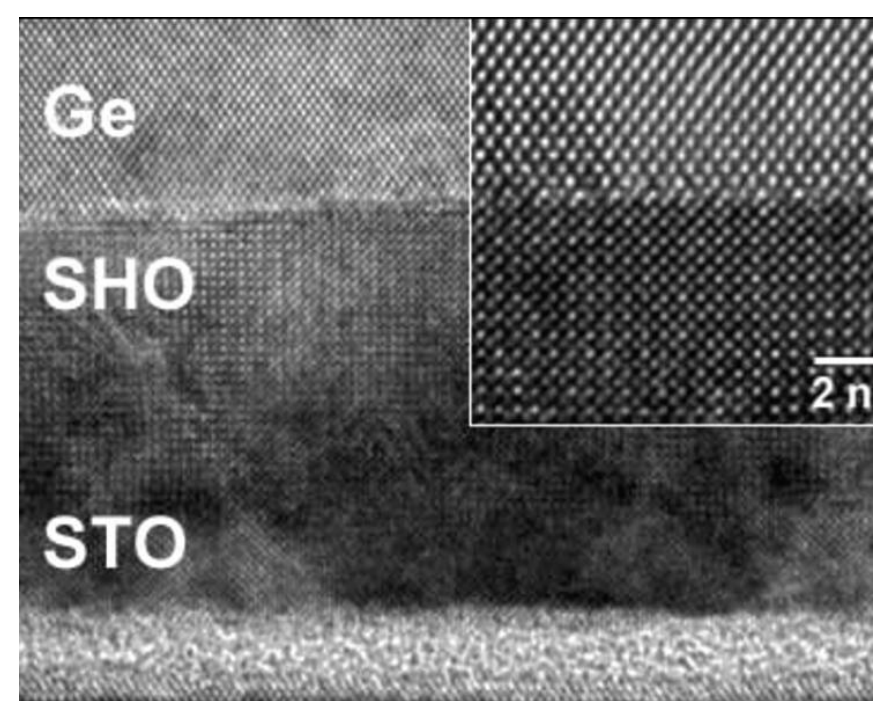

The second oxide based approach, Ge on high Ge content SGOI substrates, is relatively new but has produced encouraging results. The method usually involves using the Ge condensation technique, proposed by Tezuka et al. [67], to convert a standard SOI substrate into a SGOI substrate. In this technique, a layer of low Ge content $\mathrm{Si}_{1-x} \mathrm{Ge}_{x}(x \leq 0.15)$ is epitaxially grown on a SOI substrate. A thinner layer of $\mathrm{Si}$ is grown on this $\mathrm{SiGe}$ layer, and then the structure is cycled through oxidizing and annealing stages. Due to the preferential oxidation of $\mathrm{Si}$ over $\mathrm{Ge}$ [68], the original $\mathrm{Si}_{1-x} \mathrm{Ge}_{x}$ layer recedes at the upper and lower bounds to leave behind $\mathrm{SiO}_{2}$. After oxidation and additional annealing in $\mathrm{N}_{2}$ to homogenize the Ge distribution, a thinner layer of Ge-enriched $\mathrm{Si}_{1-x} \mathrm{Ge}_{x}$ is left sandwiched between thicker layers of $\mathrm{SiO}_{2}$ as seen in Figure 5. In the last step the top layer of $\mathrm{SiO}_{2}$ is etched with $\mathrm{HF}$ to reveal the crystalline $\mathrm{Si}_{1-x} \mathrm{Ge}_{x}$ surface. Ma et al. [65] used an optimized oxidation and annealing sequence to achieve a $\mathrm{Si}_{0.19} \mathrm{Ge}_{0.89}(16.8 \mathrm{~nm}$ thick) surface with a RMS roughness of $0.8 \mathrm{~nm}$ and a TDD 
of $1.7 \times 10^{4} \mathrm{~cm}^{-2}$. While the surface is not pure $\mathrm{Ge}$, with the high Ge fractional content and exceptional surface quality it may prove to be a suitable template for Ge growth, or strained Ge in particular. Further experiments are needed to determine if this is the case. Due to the thick layer of $\mathrm{SiO}_{2}$ under the Ge rich $\mathrm{Si}_{1-x} \mathrm{Ge}_{x}$, devices grown on this platform should be well isolated. However, Ge rich $\mathrm{Si}_{1-x} \mathrm{Ge}_{x}$ would be expected to have a bandgap near $0.8 \mathrm{eV}$, low enough to be a potential pathway for parallel conduction. Hutin et al. [8] showed this parallel conduction could be reduced by ensuring the Ge rich $\mathrm{Si}_{1-x} \mathrm{Ge}_{x}$ layer is very thin $(8 \mathrm{~nm})$. Encouragingly, they reported an $I_{\mathrm{on}} / I_{\mathrm{off}}$ ratio of approximately $10^{5}$, the one of the best reported for a pMOSFET fabricated using the Ge condensation technique. Recently, even more encouraging results have been obtained [69].

On the topic of heterogeneous integration of Ge on Si substrates, there does not yet appear to be a clear winner for the best approach. However, many promising results have been obtained so far. The challenge of $\mathrm{Ge}$ on $\mathrm{Si}$ growth remains an important and ongoing area of research to unlock the potential of Ge based devices.

Figure 5. HRTEM images of Ge-rich SiGe layer produced by the Ge condensation technique. The Ge composition is $89 \%$, reprinted with permission from [65]. Copyright 2009, Elsevier.

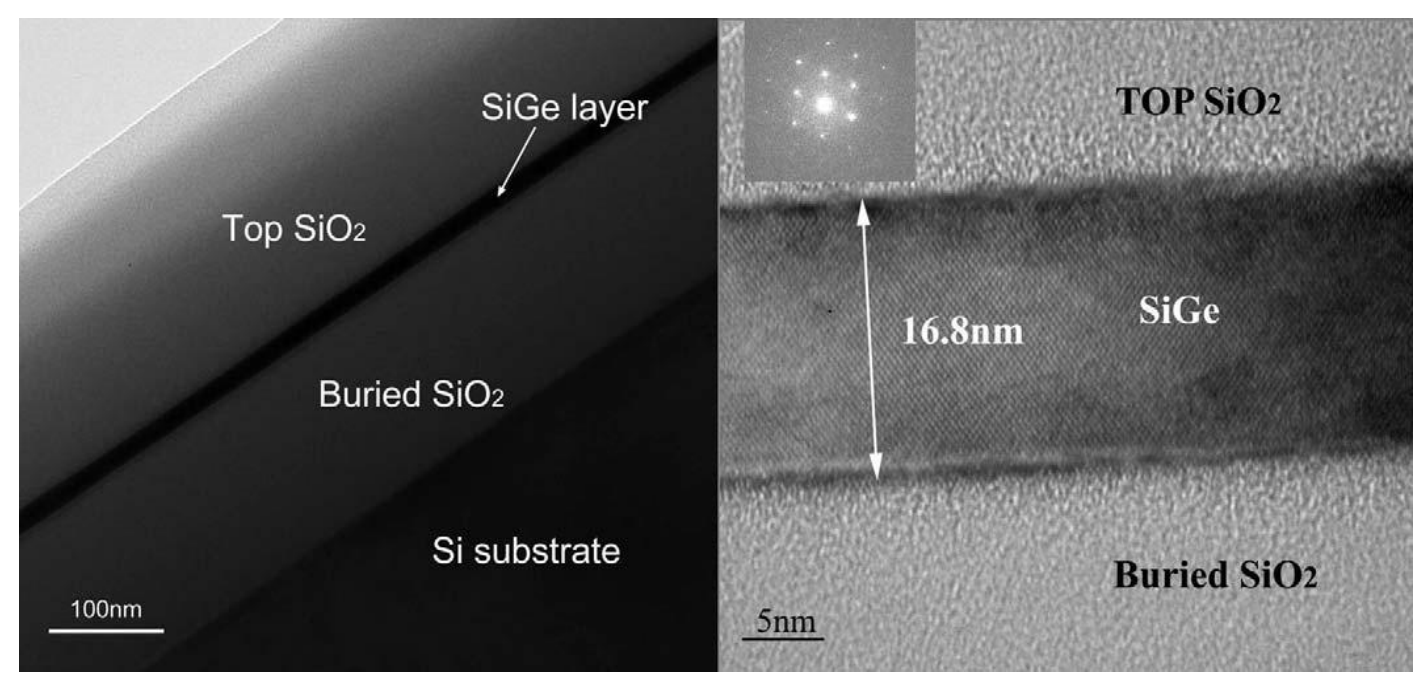

\subsection{Developing a Suitable Gate Stack}

The era of high- $\kappa$ oxides has brought with it many exciting opportunities and challenges of its own. High-k materials deposited on Ge, in particular, have poised a special challenge due the higher reactivity of $\mathrm{Ge}$ (compared to $\mathrm{Si}$ ) and the instability of the semiconductor's native oxide, a subject discussed in more detail below. A number of excellent reviews which include a discussion of high-k oxides on Ge exist currently in the literature [2,11,27-29,70-73]. However, with the rapid pace of development in this area, recent advancements merit further review.

\subsubsection{Overview}

The critical function of high-k oxides is to permit a greater physical thickness of dielectric material between the gate and channel (compared to $\mathrm{SiO}_{2}$ ) without sacrificing gate capacitance. The charge component, $Q$, of $I_{\mathrm{on}}$ is summoned by the combination of the gate voltage and gate capacitance, 
therefore a high gate capacitance is desired to increase $I_{\mathrm{on}}$. For decades this capacitance could be scaled higher by making the oxide thinner. However, there is a lower limit beyond which the oxide thickness cannot be reduced since tunneling current (leakage) increases exponentially with decreasing thickness. Oxides with a physical thickness less than $\sim 2 \mathrm{~nm}$ exhibit a unacceptably large gate leakage current leading to excessive power dissipation [74]. The gate capacitance is:

$$
C=\frac{\varepsilon_{0} k A}{t_{\mathrm{ox}}}
$$

where $A$ is the area, $k$ is the dielectric constant, $\varepsilon_{0}$ is the permittivity of free space, and $t_{\mathrm{ox}}$ is the oxide thickness. The relation above motivates the implementation of high-k materials into gate stacks. The only way to increase $C$, when $t_{\mathrm{ox}}$ cannot be reduced further, is to increase $k$ (assuming $A$ is fixed).

An important fundamental concept in discussing gate stacks is equivalent oxide thickness (EOT). Since industry used $\mathrm{SiO}_{2}$ as the gate oxide for decades, researchers and device engineers have grown accustomed to using the thickness of the $\mathrm{SiO}_{2}$ as the primary metric to gauge the strength of the gate capacitance. Today, this remains the preferred means to assess gate capacitance, even after $\mathrm{SiO}_{2}$ is no longer the primary oxide used in today's microprocessors. EOT is computed as:

$$
t_{\mathrm{eq}}=E O T=\left(\frac{3.9}{k_{\mathrm{hiK}}}\right) t_{\mathrm{hiK}}
$$

where $k_{\mathrm{hiK}}$ and $t_{\mathrm{hiK}}$ are the dielectric constant and physical thickness of the high-k material respectively. For Ge and III-V devices, the ITRS calls for a manufacturable EOT of $0.68 \mathrm{~nm}$ by 2018 [31]. This is a good target for today's research efforts. For a material with a dielectric constant of 20 , this corresponds to a physical oxide thickness of $4.36 \mathrm{~nm}$.

In truth, the description of the gate capacitance provided by Equations (2) and (3) is oversimplified. Today's deep-submicron MOSFETs have entered a regime in which quantum mechanical effects and the gate electrode (degenerately doped poly-Si) depletion depth can no longer be neglected. These effects are reviewed in number of excellent works [74-76] and are not discussed in detail here. The thin depletion region in the poly-Si and the finite displacement of the charge centroid (in the channel) from the oxide interface both contribute an additional capacitance term appearing in series with the oxide capacitance. Therefore, the gate capacitance is more accurately modeled as:

$$
\frac{1}{C}=\frac{1}{C_{\text {ox }}}+\frac{1}{C_{\text {poly }}}+\frac{1}{C_{\text {channel }}} .
$$

Then the effective capacitance thickness (ECT, also called the electrical EOT in some literature) follows as a sum of three effective thicknesses as shown below:

$$
E C T=E O T+t_{\text {poly }}+t_{\text {channel }}
$$

where $t_{\text {poly }}$ and $t_{\text {channel }}$ are the effective $\mathrm{SiO}_{2}$ equivalent thicknesses of $C_{\text {poly }}$ and $C_{\text {channel }}$ respectively. These thicknesses can each contribute few $\AA$ to the ECT. $t_{\text {poly }}$ can be reduced to about $0.5 \AA$ by replacing the poly-Si gate electrode with a metal (which is itself separate technical challenge). However, $t_{\text {channel }}$ is an intrinsic phenomenon, and likely intractable. For Ge FET devices, the ITRS calls for an ECT of $1.18 \mathrm{~nm}$ by 2018 .

High-k materials can be deposited on Ge using a variety of methods. The most common are chemical vapor deposition (CVD) [77,78], physical vapor deposition (PVD) [79], molecular beam 
deposition (MDB) [80,81], and atomic layer deposition (ALD) (two variations: thermal [82], and plasma [83]). Among these four, ALD is the most popular since it has many advantages. In addition to allowing precise thickness control on the sub-nm scale, ALD offers superior step-coverage and conformity, which is doubly important as we move into the era of multi-gate and $3 \mathrm{D}$ transistor configurations [84].

Experimentally, poor performance has been obtained when high-k oxides are deposited directly onto the Ge channel due to a high density of defects that create electronic states in the bandgap. High-k materials intrinsically have a higher concentration of defects compared to $\mathrm{SiO}_{2}$ due to their higher coordination number. Bonds in high-k materials cannot relax and rebond at defect sites as well as $\mathrm{SiO}_{2}$. The concentration of defects, particularly those near the interface, has serious consequences for channel mobility. Charge trapped in the defect centers scatter carriers in the channel leading to degradation of channel mobility [74]. To address this issue, an interfacial layer $(I L)$ is usually inserted between the high-k material and the channel. The $I L$ material generally has a much lower dielectric constant and tends to form a higher quality interface (fewer defects) with the channel material. The reduction of the defect concentration at the interface is often called surface passivation and so these layers are also called passivating layers. The metric most commonly used to judge the quality of an interface is the interface trap density $D_{\text {it }}$, which can be measured using methods reviewed in refs. $[85,86]$ (the terms interface traps, interface charge, and interface states are all used interchangeably in the literature). $D_{\mathrm{it}}$ represents the number of trapped charges per $\mathrm{cm}^{2}$ per $\mathrm{eV}$. The term $\mathrm{eV}$ appears since the energy levels of the traps are distributed across the bandgap. Therefore, trap density is reported as a function of energy level. Usually, however, only the density near the midgap is reported (unless otherwise stated).

In addition to interface passivation, a critical role of the $I L$ is to put some distance between carriers in the channel and the high-k material. This is highly desirable since high-k materials have been shown to strongly impact channel mobility even when not in direct contact with it [87]. The reasons for this are not entirely clear, however three mechanisms have been put forward (listed without details here since they are beyond the scope this this article): remote optical phonon scattering (RPS) [88,89], remote Coulomb scattering (RCS) [90,91], and remote surface roughness scattering [92,93]. Whatever the mechanism, one thing is clear, the presence of a high-quality $I L$ is critically important to preserving the intrinsically high carrier mobility of Ge in the channel. The importance of the $I L$ channel-shielding effect must be carefully balanced with the EOT cost of including the $I L$. Since $I L$ s have lower dielectric constants, they generally increase the total EOT presented by the high-k material and the $I L$ in series. This is seen from the equation for adding capacitors in series (same form as in Equation (5)). Neglecting poly-Si depletion and quantum mechanical effects we have:

$$
\frac{1}{C_{\mathrm{eq}}}=\frac{1}{C_{\mathrm{hi}-\mathrm{k}}}+\frac{1}{C_{I L}} .
$$

Combining Equations (2), (3), and (6) we can derive:

$$
E O T_{\text {total }}=\left(\frac{3.9}{k_{\mathrm{hi}-\mathrm{k}}}\right) t_{\mathrm{hi}-\mathrm{k}}+\left(\frac{3.9}{k_{I L}}\right) t_{I L}
$$

where $k_{I L}$ and $t_{I L}$ are the dielectric constant and physical thickness of the $I L$ material respectively. To further illustrate the notion of the $I L E O T$ cost, consider the following example. Consider an $I L$ 
which is $1 \mathrm{~nm}$ thick and is comprised of material with a dielectric constant of 4.5 . Assume at least $1 \mathrm{~nm} I L$ thickness is needed to give low $D_{\text {it }}$ and good channel mobility. Since a physical thickness of greater than $2 \mathrm{~nm}$ is strongly desired to suppress gate leakage, $2 \mathrm{~nm}$ of high-k material with a dielectric constant of 20 is deposited on top of the $I L$ for a total physical oxide thickness of $3 \mathrm{~nm}$. This configuration gives a total EOT of $1.26 \mathrm{~nm}$, approximately double the ITRS target indicated earlier.

The EOT cost of including an $I L$ has motivated many experiments to find alternate passivation methods, usually wet chemical approaches that do not involve a physical layer of lower $k$ material in the gate stack. These methods have generally been unsuccessful due to high $D_{\text {it }}[94,95]$. One of the primary goals of Ge gate stack research today is to simultaneously achieve low EOT, high channel mobility, and low leakage current. Such a device, however, has thus far proven to be exceedingly elusive. In fact, a general trend has been observed: as EOT is scaled down, peak channel mobility is degraded [11,74].

Another important consideration in developing gate stacks is band offsets. Both the conduction and valence band offsets (CBO and VBO respectively) must be at least $1 \mathrm{eV}$ in order to suppress Schottky emission of electrons or holes into the oxide bands (another source of leakage current) [96]. Figure 6 shows predicted Ge CBOs and VBOs with an array of common gate oxides. Almost always, the CBO is the smaller of the two offsets, so it is generally of greater interest.

The requirements from the gate oxide stack are summarized as follows:

1. Low EOT (should approach or exceed the targets set by the ITRS);

2. Low leakage current density (should not be higher than $1.5 \times 10^{-2} \mathrm{~A} / \mathrm{cm}^{2}$ ) [74];

3. Sufficient $\mathrm{CBO}$ and $\mathrm{VBO}$ (at least $1 \mathrm{eV}$ for both);

4. Low $D_{\text {it }}$ (less than $10^{11} \mathrm{~cm}^{-2} \cdot \mathrm{eV}^{-1}$ is good, greater than $10^{12} \mathrm{~cm}^{-2} \cdot \mathrm{eV}^{-1}$ is poor);

5. Minimally diminished channel mobility (although this is usually expected if $D_{\mathrm{it}}$ is low);

6. Sufficiently high dielectric breakdown electric field $\mathrm{E}_{\mathrm{BD}}[73]$;

7. Thermodynamic and kinetic stability [74].

Figure 6. Calculated band offsets of oxides on Ge, reprinted with permission from [97]. Copyright 2013, AIP Publishing LLC.

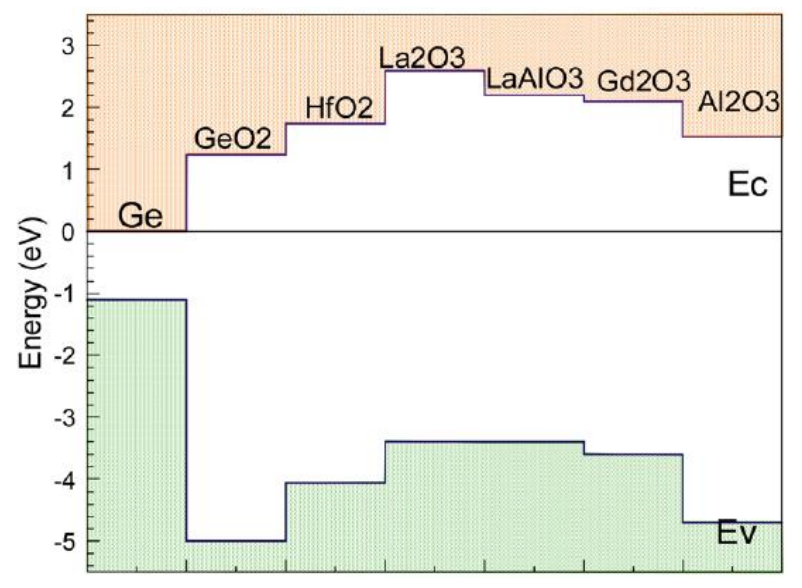

In regards to thermodynamic and kinetic stability, the parameters listed in requirements $1-6$ should be relatively immune to high temperature device processing steps (for example if the gate oxide is amorphous it must not recrystallize during processing); $400{ }^{\circ} \mathrm{C}$ Ge MOSFET process technology has 
been demonstrated [98]. Following from this process, Ge gate stacks should be able to endure thermal budgets of at least $400{ }^{\circ} \mathrm{C}$ for $30 \mathrm{~min}$. Although this is substantially less than typical thermal budgets for $\mathrm{Si}$, it remains a serious challenge since (i) key high-k oxides, such as $\mathrm{HfO}_{2}$, are known to recrystallize at $400{ }^{\circ} \mathrm{C}$ [99], (ii) $\mathrm{GeO}_{2}$ (sometimes used as an $I L$ for gate stacks on Ge) is highly unstable at this temperature (this is discussed in more detail in the next section), and (iii) Ge can interdiffuse with important high-k materials like $\mathrm{HfO}_{2}$ and $\mathrm{ZrO}_{2}$, even at temperatures as low as $300{ }^{\circ} \mathrm{C}$, contributing to higher $D_{\text {it }}$ values [71]. The most common materials used as high-k dielectrics on Ge are $\mathrm{Al}_{2} \mathrm{O}_{3}, \mathrm{HfO}_{2}$, and $\mathrm{ZrO}_{2}$. These materials have been selected since they have (i) relatively high $k$ values as shown in Table 2, (ii) sufficient $\mathrm{CBO}$ and VBO, (iii) reasonable thermodynamic and kinetic stability, and (iv) adequately high breakdown electric field. Other materials are also being investigated such as $\mathrm{Y}_{2} \mathrm{O}_{3}$ [100], $\mathrm{TiO}_{2}$ [13], and $\mathrm{La}_{2} \mathrm{O}_{3}$ [101].

With the ground work covered, the following sections will review methods for implementing an $I L$ on Ge surfaces. The role of the $I L$ is twofold: (i) passivate the surface effectively (i.e., have low $D_{\text {it }}$ and give undiminished channel mobility), and (ii) accomplish role (i) at the lowest EOT cost possible. The quality of the $I L$ layer is determined by its ability to achieve the above stringent requirement. In the case of Ge, two $I L$ approaches have emerged as the most successful over recent years: the $\mathrm{GeO}_{2}$ passivation layer [102-104], and the Si passivation layer [6,105-108]. Other approaches exist, such as surface nitridation [77], and sulfur passivation [109], however these approaches are not reviewed in this paper.

Table 2. Static dielectric constant $k$ and experimental bandgap for gate dielectrics common in the literature (reproduced from ref. [74]).

\begin{tabular}{ccc}
\hline \multicolumn{1}{c}{$\boldsymbol{k}$} & & Gap (eV) \\
\hline $\mathrm{SiO}_{2}$ & 3.9 & 9 \\
$\mathrm{Si}_{3} \mathrm{~N}_{4}$ & 7 & 5.3 \\
$\mathrm{Al}_{2} \mathrm{O}_{3}$ & 9 & 8.8 \\
$\mathrm{Ta}_{2} \mathrm{O}_{5}$ & 22 & 4.4 \\
$\mathrm{TiO}_{2}$ & 80 & 3.5 \\
$\mathrm{SrTiO}_{3}$ & 2000 & 3.2 \\
$\mathrm{ZrO}_{2}$ & 25 & 5.8 \\
$\mathrm{HfO}_{2}$ & 25 & 5.8 \\
$\mathrm{HfSiO}_{4}$ & 11 & 6.5 \\
$\mathrm{La}_{2} \mathrm{O}_{3}$ & 30 & 6 \\
$\mathrm{Y}_{2} \mathrm{O}_{3}$ & 15 & 6 \\
$\alpha-\mathrm{LaAlO}_{3}$ & 30 & 5.6 \\
\hline
\end{tabular}

Before proceeding, a word of caution must be issued to the reader. One of the greatest issues plaguing gate stack research is the lack of consensus on the procedure for measuring and extracting $D_{\text {it }}[85,86,110-113]$. Without standardization of the $D_{\text {it }}$ measurement, comparing outcomes for different gate stack approaches is difficult. This also increases the challenge in identifying a clear relationship between $D_{\text {it }}$, channel mobility, and subthreshold slope. 


\subsubsection{The $\mathrm{GeO}_{2}$ Passivation Layer}

The success of $\mathrm{Si}$ native oxide $\mathrm{SiO}_{2}$ as an interface passivation scheme for $\mathrm{Si}$ devices helped sustain the growth of the microelectronics industry for decades. This has led many to investigate using Ge's native oxide as a passivation layer in Ge devices. Ge's native oxide is usually written as $\mathrm{GeO}_{x}$. The $x$ is intended to emphasize that $\mathrm{GeO}_{2}$ is not always dominant (this is in contrast to $\mathrm{Si}$ where $\mathrm{SiO}_{2}$ is dominant). It is primarily composed of a mixture of $\mathrm{GeO}$ and $\mathrm{GeO}_{2}$ [114]. Prabhakaran et al. [115] showed that as annealing temperature is increased from $250{ }^{\circ} \mathrm{C}$ to $420{ }^{\circ} \mathrm{C}$ the $\mathrm{GeO}_{2}$ on the surface undergoes the following transformation:

$$
\mathrm{GeO}_{2}+\mathrm{Ge} \rightarrow 2 \mathrm{GeO}
$$

Furthermore, at approximately $425^{\circ} \mathrm{C}$ the $\mathrm{GeO}$ desorbs from the surface. This marks a stark contrast to the $\mathrm{SiO}_{2} / \mathrm{Si}$ interface in which the monoxide species is transformed to the dioxide species as annealing temperature approaches the oxide desorption temperature. The transformation is as follows:

$$
2 \mathrm{SiO} \rightarrow \mathrm{SiO}_{2}+\mathrm{Si}
$$

Relations (8) and (9) illustrate the distinctly thermal decomposition pathways of native oxide layers on $\mathrm{Si}$ and Ge. Additionally, $\mathrm{SiO}_{2}$ does not desorb until $760{ }^{\circ} \mathrm{C}$. By comparison to the $\mathrm{SiO}_{2} / \mathrm{Si}$ interface, the $\mathrm{GeO}_{x} / \mathrm{Ge}$ interface is far less thermodynamically stable. Ge's poor stability is exacerbated by the fact that $\mathrm{GeO}_{2}$ is soluble in water. This stark contrast in behavior helps explain why there is a substantial difference in quality (in terms of $D_{\text {it }}$ ) between the $\mathrm{SiO}_{2} / \mathrm{Si}$ and the $\mathrm{GeO}_{x} / \mathrm{Ge}$ interface. For the $\mathrm{SiO}_{2} / \mathrm{Si}$ interface a $D_{\text {it }}$ of $\sim 10^{10} \mathrm{~cm}^{-2} \cdot \mathrm{eV}^{-1}$ is routinely obtained [116]. However, for the $\mathrm{GeO}_{x} / \mathrm{Ge}$ interface, even $10^{11} \mathrm{~cm}^{-2} \cdot \mathrm{eV}^{-1}$ is very difficult to achieve [117,118]. More recently, it has been found that $\mathrm{GeO}_{2}$ can serve as an effective $I L$, and the quality of the interface is strongly dependent on how the interfacial oxide is formed [102-104]. A wide variety of oxidation methods are being explored, including thermal oxidation [12,71,102,119-130], ozone or atomic oxygen exposure [131-136], high-pressure oxidation [103,104,137], radical oxidation [138], and plasma techniques [9,14,139].

Perhaps the greatest challenge of the $\mathrm{GeO}_{2} I L$ approach is controlling (i) the thickness of the $\mathrm{GeO}_{2}$, and (ii) the diffusion of Ge into high-k material. It is desirable to keep the $\mathrm{GeO}_{2}$ no thicker than is needed to passivate the interface (to minimize the ILEOT cost). This is made more difficult by the fact that Ge has a tendency to diffuse into the high-k material [103,140]; although some materials have shown to be more resistant to Ge up-diffusion and can even act as a barrier to it $\left(\mathrm{Al}_{2} \mathrm{O}_{3}\right.$ is an example) [14]. Some works have observed that suppressing Ge interdiffusion with the high-k material results in improved performance [14,140]; suggesting defects are created during the interdiffusion process. Recently, researchers have shown some success in improving interface quality by capping the $\mathrm{GeO}_{2} / \mathrm{Ge}$ interface with diffusion resistant high-k material [13,14,138].

Recently, Zhang et al. [13] demonstrated an EOT of $0.65 \mathrm{~nm}$ using a gate stack of $\mathrm{TiO}_{2} / \mathrm{Al}_{2} \mathrm{O}_{3} / \mathrm{Ge}$. The group reported their expectation that there were a few monolayers of $\mathrm{GeO}_{2}$ at the $\mathrm{Al}_{2} \mathrm{O}_{3} / \mathrm{Ge}$ interface. Previous analysis [141] of a device made using the same process found a $D_{\text {it }}$ of about $3 \times 10^{11} \mathrm{~cm}^{-2} \cdot \mathrm{eV}^{-1}$ near the midgap. $\mathrm{TiO}_{2}$ is an attractive dielectric material since it has a very high dielectric constant of 80 . However, it is rarely used since its $\mathrm{CBO}$ on $\mathrm{Ge}$ is almost zero. $\mathrm{Al}_{2} \mathrm{O}_{3}$ on the other hand has a relatively low dielectric constant of 9 and a $\mathrm{CBO}$ of $1.58 \mathrm{eV}$ on Ge, which meets the $1 \mathrm{eV}$ minimum to suppress gate leakage. For this work, a fair leakage current of about $1 \times 10^{-2} \mathrm{~A} / \mathrm{cm}^{2}$ at 
$V_{\mathrm{g}}=-1 \mathrm{~V}$ is reported for the combined $\mathrm{TiO}_{2} / \mathrm{Al}_{2} \mathrm{O}_{3} / \mathrm{Ge}$ stack. This is notable since a very poor leakage current might be expected due to the near zero $\mathrm{CBO}$ of $\mathrm{TiO}_{2}$ on $\mathrm{Ge}$ and the $\mathrm{Al}_{2} \mathrm{O}_{3}$ layer is fairly thin $(\sim 1 \mathrm{~nm})$. Since it seems no single dielectric material can achieve everything required for an excellent gate stack, composite gate stacks provide some much needed flexibility, as well as many exciting and unexplored possibilities for Ge gate stack design.

Another recent and promising development is post-plasma oxidation. Zhang et al. [14] demonstrated an EOT of about $1 \mathrm{~nm}$ and midgap $D_{\text {it }}$ on the order of $1 \times 10^{11} \mathrm{~cm}^{-2} \cdot \mathrm{eV}^{-1}$ using a $\mathrm{Al}_{2} \mathrm{O}_{3} / \mathrm{GeO}_{x} / \mathrm{Ge}$ gate stack. What is unique about their approach is that the $\mathrm{Al}_{2} \mathrm{O}_{3}$ is deposited before the plasma oxidation. The plasma-oxidation process then grows the $\mathrm{GeO}_{x}$ at the $\mathrm{Al}_{2} \mathrm{O}_{3} / \mathrm{Ge}$ interface. Therefore, the $\mathrm{GeO}_{x}$ is confined from the very start. Since $\mathrm{Al}_{2} \mathrm{O}_{3}$ also acts as a diffusion barrier for oxygen, the group found that by controlling the thickness of the $\mathrm{Al}_{2} \mathrm{O}_{3}$ they could control the thickness of the $\mathrm{GeO}_{x}$ interfacial layer. Additionally, the group reported a clear relationship between $D_{\text {it }}$ and the thickness of the $\mathrm{GeO}_{x}$, shown in Figure 7. The group noted $D_{\text {it }}$ increased sharply if the $\mathrm{GeO}_{x} I L$ was reduced below $0.5 \mathrm{~nm}$ and also reported a high leakage current density of $\sim 1 \mathrm{~A} / \mathrm{cm}^{2}$, which is likely due to the relatively thin combined oxide thickness of $\sim 2.5 \mathrm{~nm}$.

Figure 7. (a) Thicknesses of $\mathrm{GeO}_{x}$ ILs with different $\mathrm{Al}_{2} \mathrm{O}_{3}$ thicknesses and plasma powers; (b) $D_{\text {it }}$ of $\mathrm{Au} / \mathrm{Al}_{2} \mathrm{O}_{3} / \mathrm{GeO}_{x} / \mathrm{Ge}$ MOS capacitors as a function of the $\mathrm{GeO}_{x} I L$ thickness. The $D_{\text {it }}$ reported is for $0.2 \mathrm{eV}$ above the valence band, reprinted with permission from [14]. Copyright 2012, IEEE.
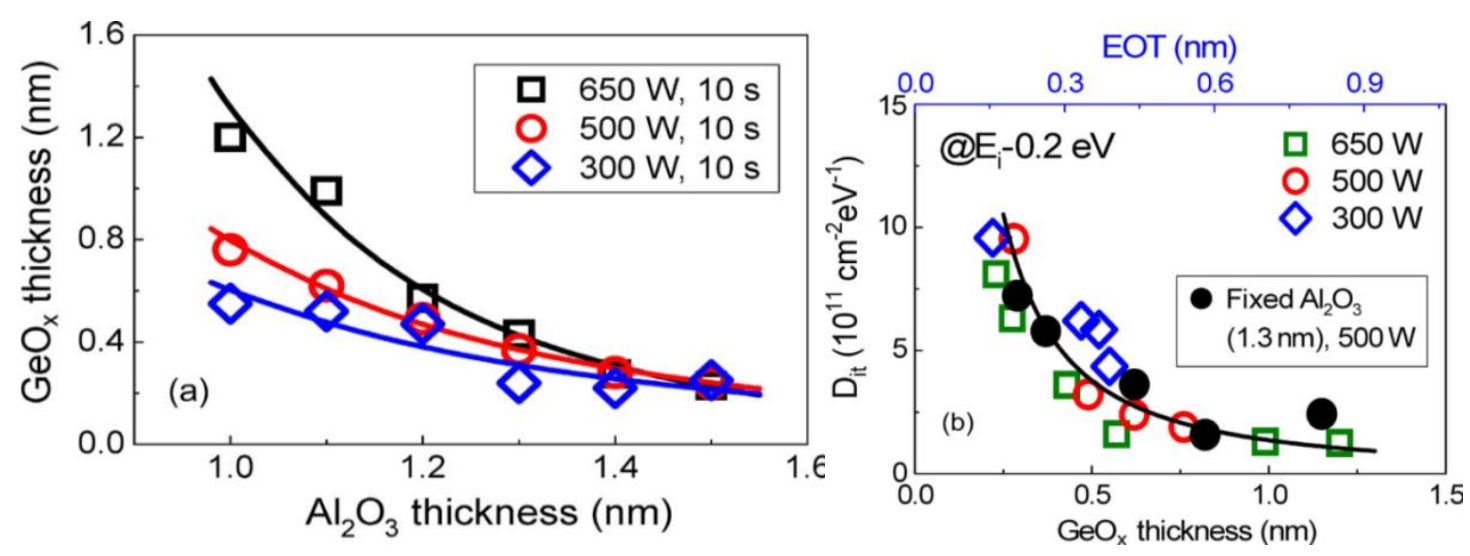

\subsubsection{The Si Passivation Layer}

The challenge of achieving high quality $\mathrm{GeO}_{x} / \mathrm{Ge}$ interfaces that are comparable to that of state-of-the-art $\mathrm{SiO}_{2} / \mathrm{Si}$ has inspired interest in the oxide/Si/Ge passivation scheme. In this approach, the $\mathrm{Si}$ is not always deliberately oxidized to form $\mathrm{SiO}_{2} / \mathrm{Si} / \mathrm{Ge}$. However, typically $2-3 \mathrm{ML}$ of $\mathrm{Si}$ is consumed during processing to form a thin $(\sim 0.5 \mathrm{~nm})$ layer of $\mathrm{SiO}_{2}$ at the $\mathrm{Si}$ surface [10,15]. The difficulty of overcoming the $4 \%$ lattice mismatch between Ge and Si has already been discussed at length. However, even extremely thin layers (less than $1 \mathrm{~nm}$ ) of Si have been shown to passivate effectively and suppress the growth of Ge's unstable native oxide [107]. This is below the critical layer thickness of Si on Ge, which has been reported to be between 1 and $2 \mathrm{~nm}$ [142]. The effectiveness of $\mathrm{Si}$ passivation has been shown to be sensitive to the Si growth parameters. It was found that diffusion of Ge into the Si layer was resulting in increased $D_{\text {it }}$ and degraded device performance. Caymax et al. [6] 
showed this Ge diffusion could be suppressed with careful selection of Si precursors and the growth temperature, with lower temperatures helping to restrict diffusion.

Intel used Si passivation to demonstrate one of the best performing Ge PMOS planar devices to date [15]. A peak hole mobility of $770 \mathrm{~cm}^{2} / \mathrm{V}$-s and equivalent oxide thickness of $1.45 \mathrm{~nm}$ was reported. Figure 8a shows a high resolution TEM image of the $\mathrm{HfO}_{2} / \mathrm{SiO}_{2} / \mathrm{Si} / \mathrm{Ge}$ gate stack. A control sample in the Intel work without the Si $I L$ showed more than a $10 x$ reduction in mobility compared to the sample with a $0.6 \mathrm{~nm} \mathrm{Si} \mathrm{cap.} \mathrm{Figure} 8 \mathrm{~b}$ shows the capacitance voltage characteristics for Ge MOSFET samples with Si caps of different thicknesses. In addition to limiting carrier spill-out, thinning the Si cap from $1.4 \mathrm{~nm}$ to $0.6 \mathrm{~nm}$ also increases the gate capacitance in the inversion region. This is expected since thinning an $I L$ comprised of a lower k material always reduces the $I L E O T$ cost. Interestingly, the group found that increasing the $\mathrm{Si}$ cap thickness beyond $0.6 \mathrm{~nm}$ resulted in a reduction in peak hole mobility, even though it also resulted in a reduction of $D_{\text {it }}$ from $9.0 \times 10^{11}$ to $1.8 \times 10^{11} \mathrm{~cm}^{-2} \cdot \mathrm{eV}^{-1}$. This is due to insufficient VBO between the Si and Ge. The potential barrier to holes is not large enough to fully confine the carriers to the high-mobility Ge channel, resulting in carrier spill-out and a reduction in overall effective mobility. This presents a critical consideration in applying the Si passivation scheme for Ge. The Si thickness must be controlled on the angstrom scale to curb carrier spill-out. Considering one lattice constant of Si is 5.431 angstroms, controlling growth at this scale is quite challenging. This task is made more difficult since some portion of this Si layer is typically consumed in the formation of $\mathrm{SiO}_{2}$. This process must be carefully controlled as well to ensure a contiguous layer of crystalline Si remains on the Ge surface. The optimum thickness of this layer appears to be approximately 6-8 ML $[10,15]$.

Figure 8. (a) Cross-sectional TEM image of a high-k metal gate stack with a thin Si cap on a Ge QWFET. Part of the Si cap is oxidized due to thermal cycles during the transistor fabrication process; (b) Capacitance-voltage characteristics of Ge pQWFETs with different Si cap thicknesses, reprinted with permission from [15]. Copyright 2010, IEEE.

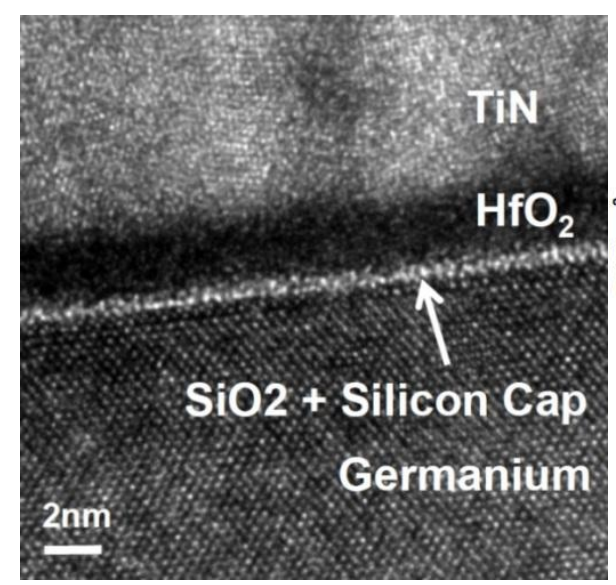

(a)

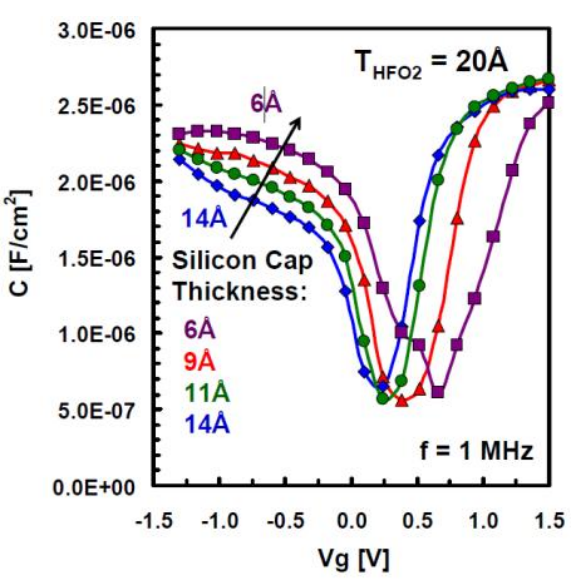

(b)

\subsection{Achieving Low-Resistance Ohmic Contacts to n-type Ge}

A key challenge in developing Ge-NMOS devices that perform comparably to the best Ge-PMOS devices today, is obtaining low-resistance ohmic contacts to n-type Ge. Low-resistance contacts are essential for a high drive current $I_{\text {on }}$. Interface states can have either donor-like or acceptor-like 
behavior. Depending on this behavior, these interface states can pin the Fermi level at the metal/semiconductor interface. For a helpful and informative review of Fermi energy pinning see [23]. By inserting an ultrathin dielectric layer between the metal and the n-doped Ge, the Fermi level can be unpinned. Fermi-level pinning close to the Ge valence band edge at the metal/Ge junction [143,144] results in a large electron Schottky barrier, which translates to a high specific contact resistivity to n-type Ge. It has been demonstrated that thin potential barriers with low CBO on Ge could enable a high tunneling current. By deliberately facilitating a high tunneling current, the effective contact resistance at the junction is reduced. This is the primary mechanism behind the metal-insulator-semiconductor (MIS) contact to Ge [145]. The insertion of thin potential barriers, such as that provided by thin layers of $\mathrm{Al}_{2} \mathrm{O}_{3}$ [146], $\mathrm{SiN}_{3}$ [147], $\mathrm{TiO}_{2}$ [145,148], $\mathrm{ZnO}$ [24], $\mathrm{Ge}_{3} \mathrm{~N}_{4}$ [149], $\mathrm{GeO}_{x}[150,151], \mathrm{MgO}[152,153]$, and $\mathrm{Y}_{2} \mathrm{O}_{3}$ [154] have been shown to reduce the Schottky barrier height as well as facilitate the unpinning of Fermi-level in n-type Ge.

It has been recently reported that the lower CBOs obtained for the crystallographic oriented $\mathrm{TiO}_{2} / \mathrm{Ge}$ system, irrespective of the Ge crystallographic orientation, presents a potential for employing a $\mathrm{TiO}_{2}$ insulating layer for MIS contact applications. Crystallographic-orientation agnostic $\mathrm{TiO}_{2}$-based MIS contacts may be particularly useful in the next generation of Ge FinFETs, where different Ge orientations can be exploited to facilitate mobility enhancement for $n$ - and $p$-channel devices [148].

\section{Opportunities}

\subsection{A Comparison: Ge versus III-V for PMOS}

High-performance n-channel InGaAs quantum well field effect transistor (QWFET) on Si has been demonstrated $[25,26,155,156]$. However, the demonstration of an equally high-performance $p$-channel QWFET within the same material system, which is needed for energy efficient complementary logic circuits, remains elusive till date due to low $\mu_{\mathrm{h}}$ in InGaAs. Strained III-Sb materials (i.e., InSb [157,158], InGaSb [159-167], and GaSb [160,165,168-173]) are potential p-channel candidates due their high hole mobilities. These materials have low in-plane heavy-hole effective mass when applying a biaxial compressive strain of 1\%-2\%. By comparison Ge has much higher bulk hole mobility $\left(\mu_{\mathrm{h}}=1900 \mathrm{~cm}^{2} / \mathrm{Vs}\right)$ even without strain. A $\mu_{\mathrm{h}}$ higher than $2400 \mathrm{~cm}^{2} / \mathrm{Vs}$ was achieved for Ge using biaxial strain, as shown in Figure 9. Theoretical investigations of hole transport in 1.5\%-2\% biaxial strained III-V semiconductors show an increase in $\mu_{\mathrm{h}}$ up to a factor of 2 over unstrained value may be possible [174-178]. However, $\mu_{\mathrm{h}}$ in $2 \%$ biaxial-strained $G e$ can be increased up to $4000 \mathrm{~cm}^{2} /$ Vs [179-181], which is significantly higher than any III-V materials. Considering several material choices and strain engineering in the channel, Ge is the best choice to obtain high $\mu_{\mathrm{h}}$ $p$-channel devices that can be heterogeneously integrated on $\mathrm{Si}$. In order to realize a Ge QWFET configuration, high bandgap barrier layers are essential in order to (i) eliminate parallel conduction to the channel $[25,26,155,156]$; (ii) provide a large VBO for hole confinement; (iii) achieve a high-quality high-k/barrier interface [182-185]; (iv) control the lattice mismatch [25,186]; (v) provide strain to the active channel; (vi) give superior interface properties; and (vii) improve ohmic contacts [187,188]. 
Figure 9. Room temperature hole mobility $v s$. sheet carrier density in biaxially compressed QWs: InSb [157,158], $\operatorname{In}_{0.4} \mathrm{Ga}_{0.6} \mathrm{Sb}, \quad \operatorname{In}_{0.35} \mathrm{Ga}_{0.65} \mathrm{As}$ [189,190], $\operatorname{In}_{0.83} \mathrm{Ga}_{0.17} \mathrm{As}$ [191], Ge [15,192-197].

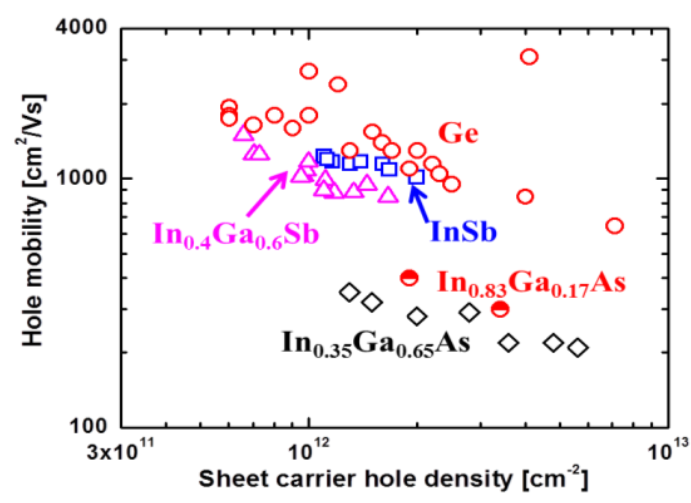

The biaxial compression in the channel causes splitting of the heavy hole $(h h)$ and light hole $(l h)$ valence bands, and causes the in-plane $h h$ mass to behave as a light hole. This results in an increase in $\mu_{\mathrm{h}}$ by reduction of $h h$ mass. By utilizing biaxial compression during the growth of $p$-channel device structures, high $\mu_{\mathrm{h}}$ (at $300 \mathrm{~K}$ ) in antimonide QWs has been achieved. $\mu_{\mathrm{h}}$ is reported as a function of sheet carrier density $\left(N_{\mathrm{s}}\right)$. In GaSb, a $\mu_{\mathrm{h}}$ of $1350 \mathrm{~cm}^{2} / \mathrm{Vs}$ was reported (at $N_{\mathrm{s}}=1.1 \times 10^{12} \mathrm{~cm}^{-2}$ with $1 \%$ strain) [168]. In InGaSb, a $\mu_{\mathrm{h}}$ of $1500 \mathrm{~cm}^{2} / \mathrm{Vs}$ was reported (at $N_{\mathrm{s}}=7 \times 10^{11} \mathrm{~cm}^{-2}$ with $2 \%$ strain) [198]. In InSb, a $\mu_{\mathrm{h}}$ of $1230 \mathrm{~cm}^{2} / \mathrm{Vs}$ was reported (at $N_{\mathrm{s}}=1.1 \times 10^{12} \mathrm{~cm}^{-2}$ with $2 \%$ strain) [157]. These works all used GaAs substrates. The reported values are far below the theoretical predictions by Hinckley and Singh [175]. Moreover, these are lower than the $\mu_{\mathrm{h}}$ of $2700-3100 \mathrm{~cm}^{2} / \mathrm{Vs}$ (at $N_{\mathrm{s}}=0.5-3 \times 10^{12} \mathrm{~cm}^{-2}$ ) that is reported for Ge QW devices, as shown in Figure 9 [192-197]. In addition, the $\mu_{\mathrm{h}}$ for arsenide-based materials (InGaAs) is much lower than for Ge or Sb-based QWs [15,173,189-191]. Figure 10 shows the in-plane $h h$ effective mass as a function of $N_{\mathrm{s}}$ in biaxially compressive strained QWs from Shubnikov-de Haas or cyclotron resonance measurements at low temperature for several materials. These results strongly suggest that the best $p$-channel material is either InSb or Ge. Utilizing Ge, with its lower lattice mismatch to $\mathrm{Si}$ (InSb is $\sim 19 \%$ versus $\sim 4 \%$ for $\mathrm{Ge}$ ), as the channel material in a QWFET configuration appears to be the most attractive option for high-mobility low-power PMOS logic.

Figure 10. Effective mass $v s$. sheet carrier density in biaxially compressed QWs.

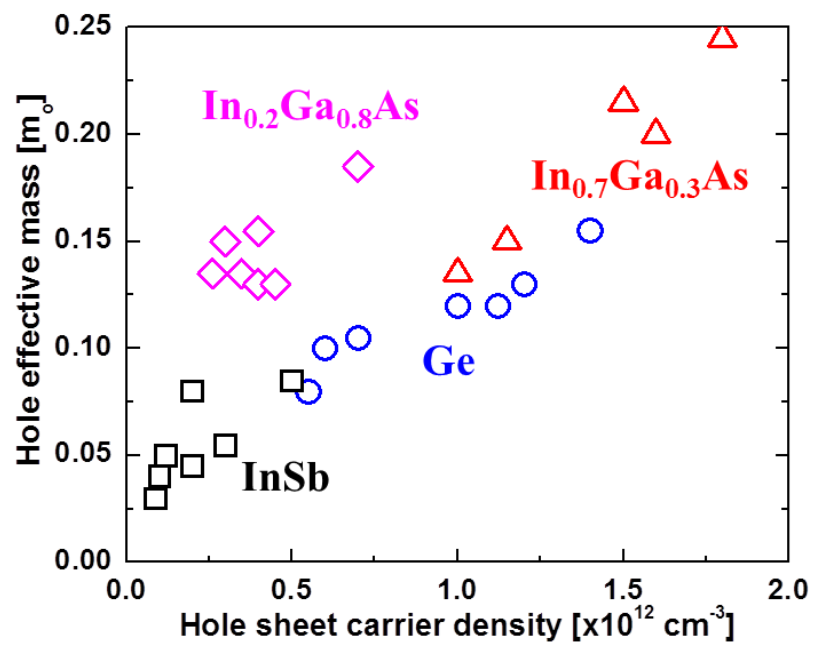




\subsection{Ge Device Architectures}

In the post-Si CMOS era, Ge is attractive due to its $2 \times$ higher $\mu_{\mathrm{n}}$ and $4 \times$ higher $\mu_{\mathrm{h}}$ (compared to $\mathrm{Si}$ ), and its lower bandgap allows for better supply voltage scalability. However, a lower supply voltage results in (i) a lower operating electric field which then lowers carrier velocity (resulting in lower $I_{\mathrm{on}}$ ), and (ii) a lower gate voltage swing (effecting the transistor's ability to switch off and resulting in increased leakage power). Therefore, new materials and innovative device architectures are required to continue transistor miniaturization. Figure 11 shows the Ge device research vehicle and compares three different device architectures: (i) conventional MOSFET, (ii) the QWFET, and (iii) metal-oxide quantum well FET (MOS-QWFET). MOS-QWFETs are a hybrid architecture in which an oxide layer replaces the upper barrier, or is deposited on top of the upper barrier, in the QWFET architecture. In the conventional MOSFET, the oxide is directly on the channel, and gate electrode is on top of the oxide. Mitard et al. [10] demonstrated a conventional Ge pMOSFET with a gate length of $65 \mathrm{~nm}$, good $I_{\mathrm{on}} / I_{\mathrm{off}}$, and scalable EOT.

Figure 11. Device research vehicle for Ge on Si for low-power logic, and comparison of MOSFET, QWFET, and MOS-QWFET structures.

(a) MOSFET

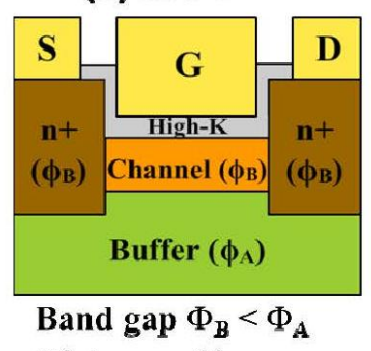

High-K on $\mathbf{S i}$ (b) QWFET

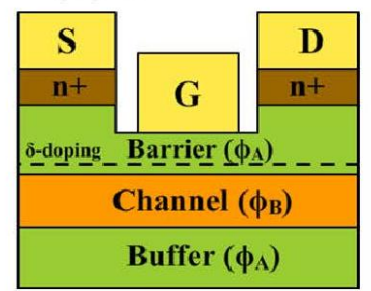

Band gap $\Phi_{\mathrm{B}}<\Phi_{\mathrm{A}} \quad$ Band gap $\Phi_{\mathrm{B}}<\Phi_{\mathrm{A}}$

Remote $\delta$-doping High-к on Si or SiGe barrier (c) Hybrid

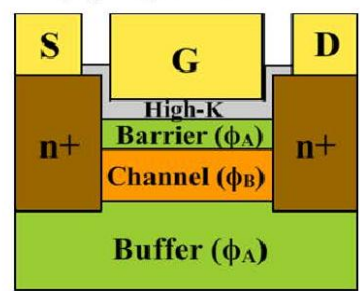

\begin{tabular}{|l|c|c|c|}
\hline & MOSFET & QWFET & Hybrid \\
\hline Mobility & + & ++ & ++ \\
\hline Gate I.eakage & + & - & + \\
\hline $\mathrm{D}_{\text {it }}$ Impact & - & + & + \\
\hline Band-to-band tunneling & + & + & + \\
\hline Self-aligned geometry & + & -- & - \\
\hline
\end{tabular}

The QWFET architecture employs a modulation doped ultra-high mobility Ge channel which facilitates efficient carrier transport at high velocity $(v(x)=\mu \times E(x))$ between the source and the drain, even at very low electric field. The channel is sandwiched by large bandgap barrier materials (SiGe or $\mathrm{Si}$ ) to reduce the leakage. In this case, the Ge channel is biaxially compressive-strained by the barrier layers. The barrier layers must also provide a VBO sufficiently high to confine holes inside the Ge channel. A modulation doping layer, separated by spacer or barrier layer, eliminates the ionized impurity scattering with holes inside the Ge channel. Therefore, the Ge channel itself is undoped, and the amount of modulation doping is carefully selected such that no charge exits underneath the gate in 
the absence of voltage applied to gate electrode. The important parameters in designing Ge QWs to enhance hole mobility are (i) the percentage of strain induced in the Ge channel, (ii) the VBO between the narrow bandgap Ge channel and the wide bandgap SiGe buffer (which determines the maximum number of holes confined in the QW), (iii) the number and effective mass of the carriers in the $l h$ and $h h$ bands, and (iv) the energy split between the $h h$ and the $l h$ bands. Using this approach, high performance Ge pQWFETs have been demonstrated at low supply voltage of $-0.5 \mathrm{~V}$ [15].

There are two varieties of QWFET architecture commonly employed in the device research community: normal and inverted. In the normal QWFET the modulation doping layer is placed above the channel. In the inverted QWFET this doping layer is placed underneath the channel. The planar (not Fin-FET or multigate design) Ge pQWFET, though it has been demonstrated, still falls short of expectations due to large separation between the source and drain metal electrodes [15]. To fabricate ultra-scaled devices below sub-22nm gate length with improved short-channel performance several issues must be overcome, namely, (i) the 1:1 ratio between the lateral to vertical scaling, (ii) the reduction in upper barrier thickness $\left(\Phi_{\mathrm{A}}\right)$ (need to be less than $2 \mathrm{~nm}$ ), and the (iii) reduction of source-to-drain spacing. The reduction in upper barrier thickness reduces the barrier resistance, which in turn improves (i) $I_{\mathrm{on}}$, (ii) switching speed, and (iii) the delay of the transistor. However, the reduction in upper barrier thickness results in increased gate leakage current for Schottky-gate (no oxide layer) QWFETs, shown in Figure 11b. The gate leakage current can be overcome with the integration of a high-k dielectric oxide, as discussed in earlier, resulting in the MOS-QWFET architecture, shown in Figure 11c, although the distinction between the QWFET and MOS-QWFET is not usually made. MOS-QWFETS exhibit significantly decreased EOT and reduced gate leakage $\left(J_{\mathrm{G}}\right)$ [15]. Therefore, the combination of the QWFET structure and the high-k gate dielectric is currently the best option for low-power logic (supply voltage of $-0.5 \mathrm{~V}$ ). In order to implement Ge MOS-QWFETs in future high-performance logic applications, scalability (both in terms of physical gate length and the overall footprint) is mandatory. A thinner upper barrier is needed for ultra-scaled buried-channel devices. Due to this stringent requirement, the modulation doping layer sometimes placed underneath the channel, resulting in the inverted QWFET structure. This results in a more scalable device with faster switching, lower dynamic power consumption and minimized $I_{\text {off }}[15]$.

\subsection{State-of-the-Art Ge MOSFETs and MOS-QWFETS}

Figure 12a,b show the cross-sectional TEM micrograph of a conventional surface channel $65 \mathrm{~nm}$ Ge pMOSFET [10] and the Ge pMOS-QWFET with in-situ boron-doped SiGe raised source/drain [15], respectively. Figure 13a shows the transfer characteristics of a $65 \mathrm{~nm}$ Ge pMOSFET at low and high $V_{\mathrm{DS}}$ with superior reproducibility [10]. Figure 13b shows the transfer characteristics for a $100 \mathrm{~nm} \mathrm{Ge}$ QWFET at $V_{\mathrm{DS}}=-0.05 \mathrm{~V}$ (open circle) and $-0.5 \mathrm{~V}$ (solid circle) [15]. The QWFET device exhibits a subthreshold slope (SS) of $97 \mathrm{mV} / \mathrm{dec}$ enabled by the phosphorus junction layer underneath the channel, which suppresses parallel conduction through the SiGe buffer as reported in [15]. Figure 14 compares saturation current ( $I_{\mathrm{dsat}}$, also called $\left.I_{\mathrm{on}}\right) v s$. off-state leakage current $\left(I_{\mathrm{off}}\right)$ characteristics of $65 \mathrm{~nm}$ Ge pMOSFET [10], $40 \mathrm{~nm} \mathrm{InSb} \mathrm{QWFET} \mathrm{[157],} \mathrm{and} 100 \mathrm{~nm} \mathrm{Ge}$ MOS-QWFET [15] at supply voltage of $-0.5 \mathrm{~V}$. The Ge MOS-QWFETs exhibited $2 \times I_{\text {dsat }}$ for the same $I_{\text {off }}$, demonstrating its potential for PMOS on a Si substrate. However, shorter gate length $(<50 \mathrm{~nm}) \mathrm{Ge}$ QWFETs in a planar configuration will be a significant challenge due to the self-aligned 
gate architecture needed for scalable enhancement mode devices for low power and high-speed digital logic applications.

Figure 12. (a) Cross-sectional TEM micrograph of a $65 \mathrm{~nm} \mathrm{Ge-pMOSFET,} \mathrm{reprinted} \mathrm{with}$ permission from [10]. Copyright 2008, IEEE; (b) Cross-sectional TEM micrograph of a Ge pQWFET, reprinted with permission from [15]. Copyright 2010, IEEE. Note the raised source/drain (RSD) in the QWFET which allows for reduction/removal of the S/D implantation.
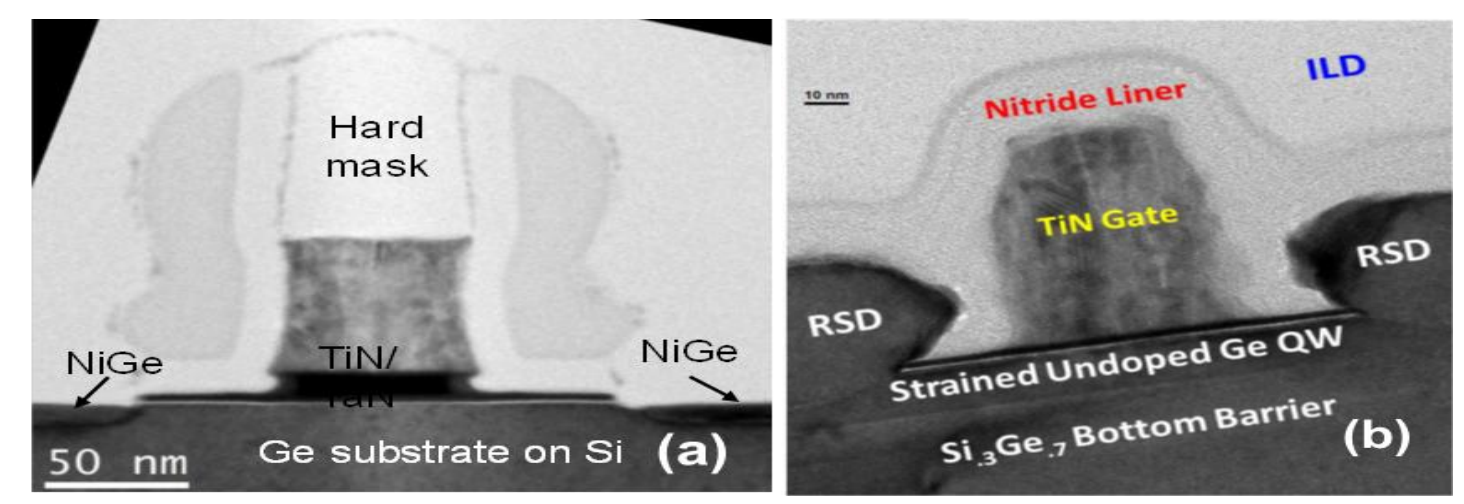

Figure 13. Transfer characteristics of measured (a) $65 \mathrm{~nm} \mathrm{Ge} \mathrm{pMOSFET} \mathrm{at} \mathrm{low} \mathrm{and} \mathrm{high}$ $\mathrm{V}_{\mathrm{DS}}$ with superior reproducibility, reprinted with permission from [10]. Copyright 2008, IEEE; (b) $100 \mathrm{~nm}$ Ge pQWFET at VDS $=-0.05 \mathrm{~V}$ (open circle) and $-0.5 \mathrm{~V}$ (solid circle). The device exhibits a subthreshold slope (SS) of $97 \mathrm{mV} / \mathrm{dec}$ enabled by the phosphorus junction layer underneath the channel, which suppresses parallel conduction through the SiGe buffer, reprinted with permission from [15]. Copyright 2010, IEEE.
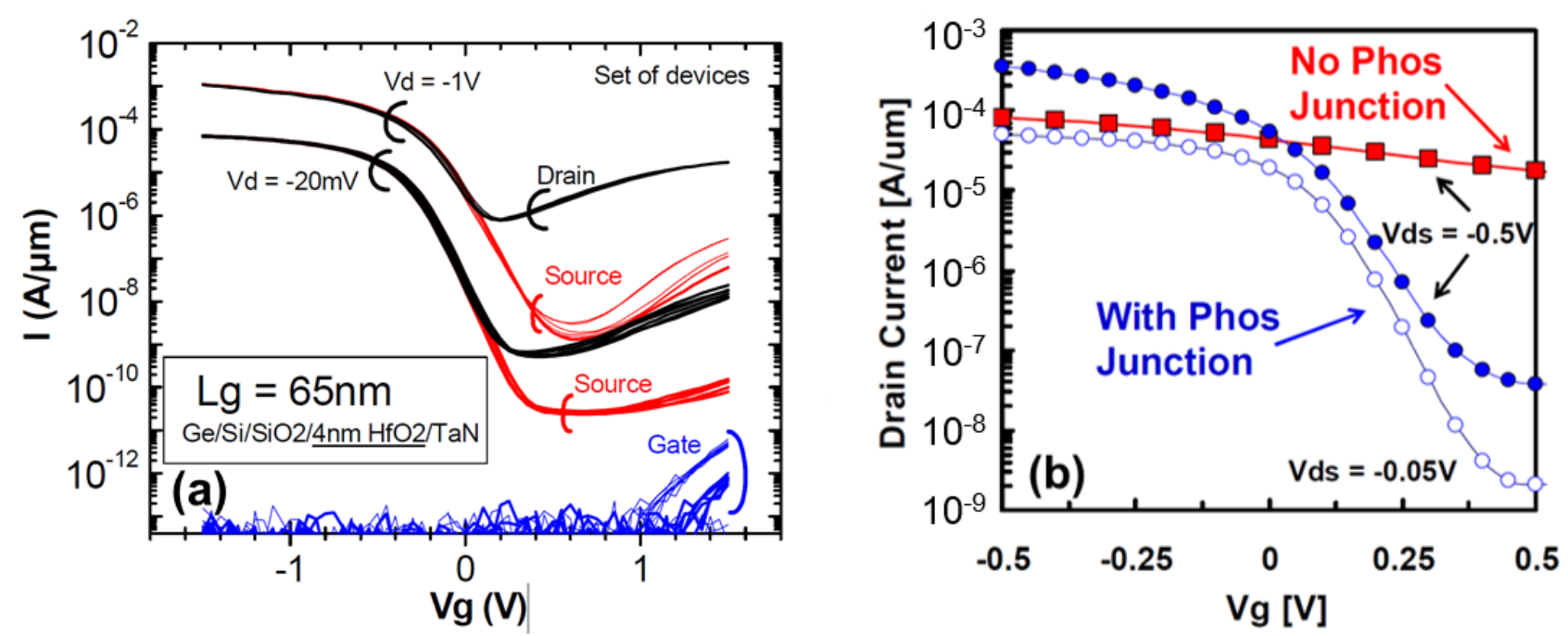

Furthermore, a list of well-established device metrics used for benchmarking logic transistors is essential [199]. These includes (a) CV/I delay as a function of transistor channel length for studying intrinsic speed of the proposed technology, (b) energy-delay product as a function of transistor channel length for optimizing switching energy and power consumption, (c) SS and drain-induced barrier lowering behavior as a function of transistor channel length which is critical for establishing the scalability, and (d) intrinsic delay $\mathrm{CV} / \mathrm{I}$ vs. $I_{\mathrm{on}} / I_{\text {off }}$ ratio to understand the performance and leakage 
tradeoff. At present, $p$-channel Ge MOS-QWFETs show great promise. Co-integration with $n$-channel InGaAs based MOS-QWFETs, as shown in Figure 15, offers an attractive pathway to extreme-high mobility CMOS. Ge based FinFET technology has recently been demonstrated [69,200]. Very recently, a Ge FinFET device obtained record $I_{\mathrm{on}} / I_{\text {off }}$ characteristics with a highly scaled gate length and EOT of $35 \mathrm{~nm}$ and $0.8 \mathrm{~nm}$ respectively, representing a considerable breakthrough [200].

Figure 14. Benchmarking relation $\left(I_{\mathrm{off}} v s . I_{\mathrm{dsat}}\right)$ of $65 \mathrm{~nm} \mathrm{Ge}$ pMOSFET, $40 \mathrm{~nm}$ InSb QWFET and $100 \mathrm{~nm}$ Ge QWFET at a supply voltage of $0.5 \mathrm{~V}$. The Ge QWFET demonstrated the highest ON current at a given OFF-state leakage current, reprinted with permission from [2]. Copyright 2011, Nature Publishing Group.

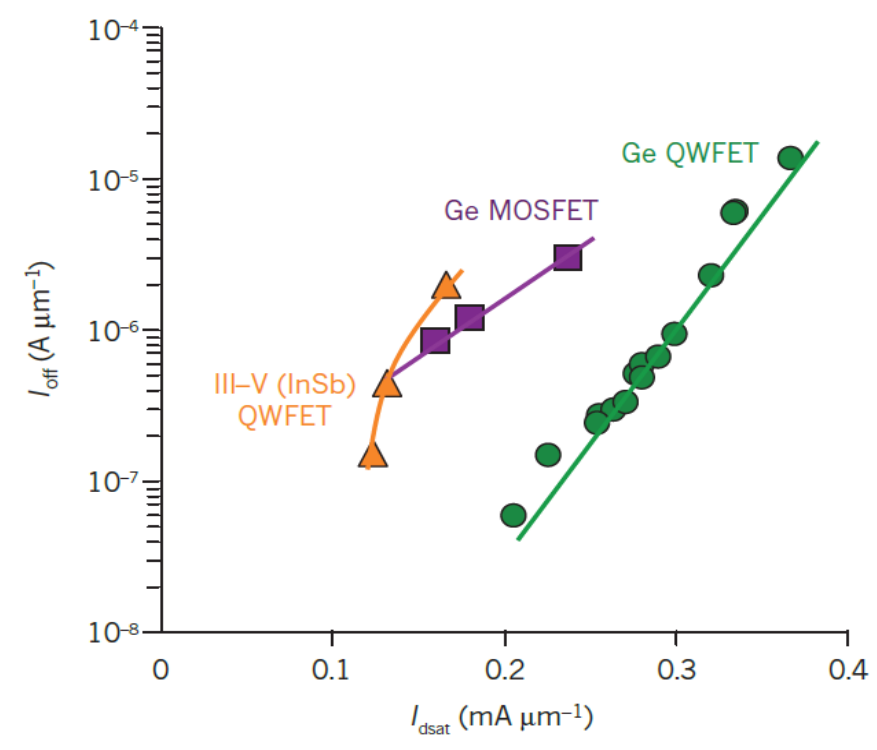

Figure 15. Schematic of extremely-high mobility NMOS and PMOS co-integrated for complete CMOS heterogeneously integrated on $\mathrm{Si}$.

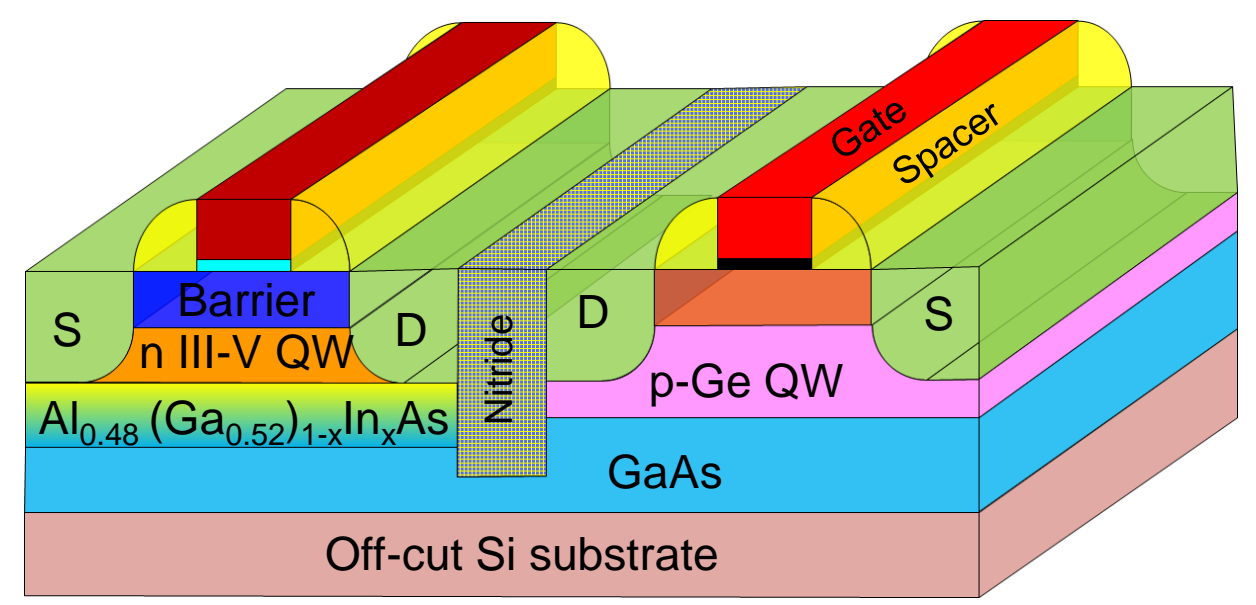

\section{Conclusions and Outlook}

In summary, germanium based $p$-channel devices are beginning to show signs of readiness for production, especially in light of recent breakthroughs [200]. Ge $n$-channel devices continue to lag behind, but are also making progress due to improved ohmic contacts [24,145]. The dual-Ge path for post-Si CMOS is becoming an increasingly viable option. However, to achieve the highest 
performance possible, the III-V/Ge path (NMOS/PMOS) is the most attractive (as shown in Figure 15), although it comes at the cost of increased processing complexity.

Five categories of buffer architectures for heterogeneous integration on Si have been discussed. Among them, the Ge condensation method has produced promising initial data, but now must demonstrate suppression of parallel conduction, and high $I_{\mathrm{on}} / I_{\text {off }}$ ratios at scaled gate lengths. The low-temperature/high-temperature method combined with hydrogen annealing has also shown good results and now must also demonstrate that parallel conduction can be controlled to achieve low $\mathrm{I}_{\text {off }}$ currents and high $I_{\mathrm{on}} / I_{\mathrm{off}}$ ratios. The SME method also offers an interesting alternative approach for $\mathrm{Ge} / \mathrm{Si}$ heteroepitaxy, especially if the matter of unintentional background doping can be resolved. The ART approach has been shown to produce narrow regions of excellent quality Ge, and has already been used in a commercial product [61].

Ge gate stacks have made tremendous strides thanks to many years of international collaborative effort. Two passivation schemes have emerged from over a decade of research. $\mathrm{GeO}_{x}$ passivation has shown it can deliver interfacial layers with $D_{\text {it }}$ comparable to today's state-of-the-art Si devices. These methods must now (i) be demonstrated at scaled gate lengths, and (ii) focus on improving the thermodynamic stability and reliability characteristics of these gate stacks. The approach of capping the $\mathrm{GeO}_{x}$ with a layer of diffusion-resistant high-k material is an excellent step in achieving this.

The metal-insulator-semiconductor (MIS) approach has shown it can effectively reduce the contact resistance to n-type Ge. These $\mathrm{n}-\mathrm{Ge}$ low resistance ohmic contacts are needed to develop Ge NMOS devices which can perform on par with Ge PMOS devices already established. If comparably performing Ge NMOS and PMOS can be obtained at scaled gate lengths it unlocks the dual-Ge CMOS path, which will substantially simplify device processing procedures (resulting in reduced costs).

The advantages of Ge over III-V for $p$-channel devices have been reviewed. As a result of many years data-driven analysis, there appears to be strong consensus in the device research community that $\mathrm{Ge}$ is the best alternative for PMOS devices (if current strained-Si PMOS technology stalls). InSb appears to be the best III-V alternative, however its lattice mismatch with $\mathrm{Si}$ is 19\%, requiring more complex and expensive buffer architectures compared to Ge (4\% lattice mismatch).

Ge device architectures have also been reviewed, along with important design considerations for Ge MOSFETs and QWFETs. The hybrid architecture, the MOS-QWFET, shows clear advantages in terms of higher $I_{\mathrm{on}}$ and lower $I_{\mathrm{off}}$. However, these devices are also difficult to scale down to gate lengths of less than $50 \mathrm{~nm}$ due to the challenge of developing a self-aligned gate. New Ge FinFET devices have shown they can overcome this limitation.

\section{Acknowledgments}

The author Patrick Goley gratefully acknowledges support from the National Science Foundation Graduate Research Fellowship under Grant No. DGE 0822220, and the Virginia Space Grant Consortium.

\section{Author Contributions}

Patrick Goley wrote and edited the manuscript. Mantu Hudait supervised the project, contributed sections 2.3, 3.1-3.3, and edited the manuscript. 


\section{Conflicts of Interest}

The authors declare no conflict of interest.

\section{References}

1. Del Alamo, J.A. Nanometre-scale electronics with III-V compound semiconductors. Nature 2011, 479, 317-323.

2. Pillarisetty, R. Academic and industry research progress in germanium nanodevices. Nature 2011, 479, 324-328.

3. Kobayashi, M.; Mitard, J.; Irisawa, T.; Hoffmann, T.; Meuris, M.; Saraswat, K.; Nishi, Y.; Heyns, M. On the High-Field Transport and Uniaxial Stress Effect in Ge PFETs. IEEE Trans. Electron Devices 2011, 58, 384-391.

4. Lin, D.; Brammertz, G.; Sioncke, S.; Fleischmann, C.; Delabie, A.; Martens, K.; Bender, H.; Conard, T.; Tseng, W.; Lin, J. Enabling the high-performance InGaAs/Ge CMOS: A common gate stack solution. In Proceedings of the IEEE International Electron Devices Meeting (IEDM), Baltimore, MD, USA, 7-9 December 2009.

5. Heyns, M.; Alian, A.; Brammertz, G.; Caymax, M.; Chang, Y.; Chu, L.; De Jaeger, B.; Eneman, G.; Gencarelli, F.; Groeseneken, G. Advancing CMOS beyond the Si roadmap with Ge and III/V devices. In Proceedings of the IEEE International Electron Devices Meeting (IEDM), Washington, DC, USA, 5-7 December 2011.

6. Caymax, M.; Leys, F.; Mitard, J.; Martens, K.; Yang, L.; Pourtois, G.; Vandervorst, W.; Meuris, M.; Loo, R. The influence of the epitaxial growth process parameters on layer characteristics and device performance in Si-passivated Ge pMOSFETs. J. Electrochem. Soc. 2009, 156, H979-H985.

7. Ho, B.; Nuo, X.; Tsu-Jae King, L. pMOSFET Performance Enhancement with Strained $\mathrm{Si}_{1-x} \mathrm{Ge}_{x}$ Channels. IEEE Trans. Electron Devices 2012, 59, 1468-1474.

8. Hutin, L.; Le Royer, C.; Damlencourt, J.-F.; Hartmann, J.-M.; Grampeix, H.; Mazzocchi, V.; Tabone, C.; Previtali, B.; Pouydebasque, A.; Vinet, M. GeOI pMOSFETs scaled down to 30-nm gate length with record off-state current. IEEE Electron Device Lett. 2010, 31, 234-236.

9. Li-Jung, L.; Kuei-Shu, C.-L.; Chung-Hao, F.; Ting-Ching, C.; Jen-Wei, C.; Chen-Chien, L.; Chun-Chang, L.; Tien-Ko, W. Ultralow EOT and high mobility Ge pMOSFETs with in-situ $\mathrm{H}_{2} \mathrm{O}$ plasma grown $\mathrm{GeO}_{2}$ and $\mathrm{HfON}$ gate dielectric. In Proceedings of the International Symposium on VLSI Technology, Systems, and Applications (VLSI-TSA), Hsinchu, Taiwan, 22-24 April 2013.

10. Mitard, J.; De Jaeger, B.; Leys, F.; Hellings, G.; Martens, K.; Eneman, G.; Brunco, D.; Loo, R.; Shamiryan, D.; Vandeweyer, T. Record ION/IOFF performance for $65 \mathrm{~nm}$ Ge pMOSFET and novel Si passivation scheme for improved EOT scalability. In Proceedings of the IEEE International Electron Devices Meeting (IEDM), San Francisco, CA, USA, 15-17 December 2008.

11. Simoen, E.; Mitard, J.; Hellings, G.; Eneman, G.; De Jaeger, B.; Witters, L.; Vincent, B.; Loo, R.; Delabie, A.; Sioncke, S.; et al. Challenges and opportunities in advanced Ge pMOSFETs. Mater. Sci. Semicond. Process. 2012, 15, 588-600.

12. Xie, R.; Phung, T.H.; He, W.; Yu, M.; Zhu, C. Interface-Engineered High-Mobility High-k/Ge pMOSFETs With 1-nm Equivalent Oxide Thickness. Electron Devices IEEE Trans. 2009, 56, 1330-1337. 
13. Zhang, L.; Gunji, M.; Thombare, S.; McIntyre, P.C. EOT Scaling of $\mathrm{TiO}_{2} / \mathrm{Al}_{2} \mathrm{O}_{3}$ on Germanium pMOSFETs and Impact of Gate Metal Selection. IEEE Electron Device Lett. 2013, 34, 732-734.

14. Zhang, R.; Iwasaki, T.; Taoka, N.; Takenaka, M.; Takagi, S. High-Mobility Ge pMOSFET With 1-nm EOT $\mathrm{Al}_{2} \mathrm{O}_{3} / \mathrm{GeO}_{x} / \mathrm{Ge}$ Gate Stack Fabricated by Plasma Post Oxidation. IEEE Trans. Electron Devices 2012, 59, 335-341.

15. Pillarisetty, R.; Chu-Kung, B.; Corcoran, S.; Dewey, G.; Kavalieros, J.; Kennel, H.; Kotlyar, R.; Le, V.; Lionberger, D.; Metz, M. High mobility strained germanium quantum well field effect transistor as the p-channel device option for low power $\left(V_{\mathrm{cc}}=0.5 \mathrm{~V}\right) \mathrm{III}-\mathrm{V}$ CMOS architecture. In Proceedings of the IEEE International Electron Devices Meeting (IEDM), San Francisco, CA, USA, 6-8 December 2010.

16. Krishnamohan, T.; Kim, D.; Nguyen, C.D.; Jungemann, C.; Nishi, Y.; Saraswat, K.C. High-mobility low band-to-band-tunneling strained-germanium double-gate heterostructure FETs: Simulations. IEEE Trans. Electron Devices 2006, 53, 1000-1009.

17. Krishnamohan, T.; Krivokapic, Z.; Uchida, K.; Nishi, Y.; Saraswat, K.C. High-mobility ultrathin strained Ge MOSFETs on bulk and SOI with low band-to-band tunneling leakage: Experiments. IEEE Trans. Electron Devices 2006, 53, 990-999.

18. Chleirigh, C.N.; Theodore, N.D.; Fukuyama, H.; Mure, S.; Ehrke, H.-U.; Domenicucci, A.; Hoyt, J.L. Thickness dependence of hole mobility in ultrathin SiGe-channel p-MOSFETs. Electron Devices IEEE Trans. 2008, 55, 2687-2694.

19. Eneman, G.; Witters, L.; Collaert, N.; Mitard, J.; Hellings, G.; Yamaguchi, S.; De Keersgieter, A.; Hikavyy, A.; Vincent, B.; Favia, P.; et al. Stress Techniques in Advanced Transistor Architectures: Bulk FinFETs and Implant-Free Quantum Well Transistors. ECS Trans. 2012, 45, 235-246.

20. Kuhn, K.J. Considerations for Ultimate CMOS Scaling. IEEE Trans. Electron Devices 2012, 59, 1813-1828.

21. Caymax, M.; Eneman, G.; Bellenger, F.; Merckling, C.; Delabie, A.; Wang, G.; Loo, R.; Simoen, E.; Mitard, J.; De Jaeger, B.; et al. Germanium for advanced CMOS anno 2009: A SWOT analysis. In Proceedings of the IEEE International Electron Devices Meeting (IEDM), Baltimore, MD, USA, 7-9 December 2009.

22. Kuzum, D.; Krishnamohan, T.; Nainani, A.; Sun, Y.; Pianetta, P.A.; Wong, H.-S.; Saraswat, K.C. High-mobility Ge N-MOSFETs and mobility degradation mechanisms. IEEE Trans. Electron Devices 2011, 58, 59-66.

23. Dimoulas, A.; Toriumi, A.; Mohney, S.E. Source and drain contacts for germanium and III-V FETs for digital logic. MRS Bull. 2009, 34, 522-529.

24. Manik, P.P.; Mishra, R.K.; Kishore, V.P.; Ray, P.; Nainani, A.; Huang, Y.-C.; Abraham, M.C.; Ganguly, U.; Lodha, S. Fermi-level unpinning and low resistivity in contacts to n-type Ge with a thin ZnO interfacial layer. Appl. Phys. Lett. 2012, 101, 182105:1-182105:5.

25. Hudait, M.K.; Dewey, G.; Datta, S.; Fastenau, J.M.; Kavalieros, J.; Liu, W.K.; Lubyshev, D.; Pillarisetty, R.; Rachmady, W.; Radosavljevic, M.; et al. Heterogeneous integration of enhancement mode $\operatorname{In}_{0.7} \mathrm{Ga}_{0.3}$ As quantum well transistor on silicon substrate using thin ( $2 \mathrm{um}$ ) composite buffer architecture for high-speed and low-voltage (0.5V) logic applications. In Proceedings of the IEEE International Electron Devices Meeting (IEDM), Washington, DC, USA, 10-12 December 2007. 
26. Radosavljevic, M.; Chu-Kung, B.; Corcoran, S.; Dewey, G.; Hudait, M.K.; Fastenau, J.M.; Kavalieros, J.; Liu, W.K.; Lubyshev, D.; Metz, M.; et al. In advanced high-K gate dielectric for high-performance short-channel $\mathrm{In}_{0.7} \mathrm{Ga}_{0.3} \mathrm{As}$ quantum well field effect transistors on silicon substrate for low power logic applications. In Proceedings of the Electron Devices Meeting (IEDM), 2009 IEEE International, Baltimore, MD, USA, 7-9 December 2009.

27. Kamata, Y. High-k/Ge MOSFETs for future nanoelectronics. Mater. Today 2008, 11, 30-38.

28. Shang, H.; Frank, M.M.; Gusev, E.P.; Chu, J.O.; Bedell, S.W.; Guarini, K.W.; Ieong, M. Germanium channel MOSFETs: Opportunities and challenges. IBM J. Res. Dev. 2006, 50, 377-386.

29. Brunco, D.; De Jaeger, B.; Eneman, G.; Mitard, J.; Hellings, G.; Satta, A.; Terzieva, V.; Souriau, L.; Leys, F.; Pourtois, G. Germanium MOSFET devices: Advances in materials understanding, process development, and electrical performance. J. Electrochem. Soc. 2008, 155, H552-H561.

30. Claeys, C.; Mitard, J.; Hellings, G.; Eneman, G.; De Jaeger, B.; Witters, L.; Loo, R.; Delabie, A.; Sioncke, S.; Caymax, M.; et al. Status and Trends in Ge CMOS Technology. ECS Trans. 2013, 54, 25-37.

31. International Roadmap for Semiconductors. Available online: http://www.itrs.net/home.html (accessed on 5 January 2014).

32. Eaglesham, D.; Cerullo, M. Dislocation-free stranski-krastanow growth of Ge on Si (100). Phys. Rev. Lett. 1990, 64, 1943-1946.

33. LeGoues, F.; Copel, M.; Tromp, R. Microstructure and strain relief of Ge films grown layer by layer on Si(001). Phys. Rev. B 1990, 42, doi:10.1103/PhysRevB.42.11690.

34. Hudait, M.K.; Zhu, Y.; Jain, N.; Hunter, J.L. Structural, morphological, and band alignment properties of GaAs/Ge/GaAs heterostructures on (100), (110), and (111) A GaAs substrates. J. Vac. Sci. Technol. B 2013, 31, 011206:1-011206:14.

35. Fang, S.F.; Adomi, K.; Iyer, S.; Morkoc, H.; Zabel, H.; Choi, C.; Otsuka, N. Gallium arsenide and other compound semiconductors on silicon. J. Appl. Phys. 1990, 68, R31-R58.

36. Nayfeh, A.; Chui, C.O.; Yonehara, T.; Saraswat, K.C. Fabrication of high-quality p-MOSFET in Ge grown heteroepitaxially on Si. Electron Device Lett. IEEE 2005, 26, 311-313.

37. Nayfeh, A.; Chui, C.O.; Saraswat, K.C.; Yonehara, T. Effects of hydrogen annealing on heteroepitaxial-Ge layers on Si: Surface roughness and electrical quality. Appl. Phys. Lett. 2004, 85, 2815-2817.

38. Choi, D.; Ge, Y.; Harris, J.S.; Cagnon, J.; Stemmer, S. Low surface roughness and threading dislocation density Ge growth on $\mathrm{Si}(001)$. J. Cryst. Growth 2008, 310, 4273-4279.

39. Luo, G.-L.; Hsieh, Y.-C.; Chang, E.Y.; Pilkuhn, M.H.; Chien, C.-H.; Yang, T.-H.; Cheng, C.-C.; Chang, C.-Y. High-speed GaAs metal gate semiconductor field effect transistor structure grown on a composite $\mathrm{Ge} / \mathrm{Ge}_{x} \mathrm{Si}_{1-\chi} \mathrm{Si}$ substrate. J. Appl. Phys. 2007, 101, 084501:1-084501:6.

40. Currie, M.T.; Samavedam, S.B.; Langdo, T.A.; Leitz, C.W.; Fitzgerald, E.A. Controlling threading dislocation densities in Ge on Si using graded SiGe layers and chemical-mechanical polishing. Appl. Phys. Lett. 1998, 72, 1718-1720.

41. Wietler, T.; Bugiel, E.; Hofmann, K. Surfactant-mediated epitaxy of relaxed low-doped Ge films on Si(001) with low defect densities. Appl. Phys. Lett. 2005, 87, 182102. 
42. Park, J.-S.; Curtin, M.; Hydrick, J.; Bai, J.; Li, J.-T.; Cheng, Z.; Carroll, M.; Fiorenza, J.; Lochtefeld, A. Low-defect-density Ge epitaxy on Si (001) using aspect ratio trapping and epitaxial lateral overgrowth. Electrochem. Solid State Lett. 2009, 12, H142-H144.

43. Suh, J.; Nakane, R.; Taoka, N.; Takenaka, M.; Takagi, S. Highly strained-SiGe-on-insulator $p$-channel metal-oxide-semiconductor field-effective transistors fabricated by applying $\mathrm{Ge}$ condensation technique to strained-Si-on-insulator substrates. Appl. Phys. Lett. 2011, 99, 142108:1-142108:3.

44. Norga, G.J.; Marchiori, C.; Guiller, A.; Locquet, J.P.; Rossel, C.; Siegwart, H.; Caimi, D.; Fompeyrine, J.; Conard, T. Phase of reflection high-energy electron diffraction oscillations during (Ba,Sr)O epitaxy on $\mathrm{Si}(100)$ : A marker of Sr barrier integrity. Appl. Phys. Lett. 2005, 87, 262905:1-262905:3.

45. Rossel, C.; Mereu, B.; Marchiori, C.; Caimi, D.; Sousa, M.; Guiller, A.; Siegwart, H.; Germann, R.; Locquet, J.P.; Fompeyrine, J.; et al. Field-effect transistors with $\mathrm{SrHfO}_{3}$ as gate oxide. Appl. Phys. Lett. 2006, 89, 053506:1-053506:3.

46. Seo, J.W.; Dieker, C.; Tapponnier, A.; Marchiori, C.; Sousa, M.; Locquet, J.P.; Fompeyrine, J.; Ispas, A.; Rossel, C.; Panayiotatos, Y.; Sotiropoulos, A.; et al. Epitaxial germanium-on-insulator grown on (001) Si. Microelectron. Eng. 2007, 84, 2328-2331.

47. Wang, J.; Lee, S. Ge-Photodetectors for Si-Based Optoelectronic Integration. Sensors 2011, 11, 696-718.

48. Michel, J.; Liu, J.; Kimerling, L.C. High-performance Ge-on-Si photodetectors. Nat. Photonics 2010, 4, 527-534.

49. Yu, H.-Y.; Park, J.-H.; Okyay, A.K.; Saraswat, K.C. Selective-Area High-Quality Germanium Growth for Monolithic Integrated Optoelectronics. Electron Device Lett. IEEE 2012, 33, 579-581.

50. Hartmann, J.; Damlencourt, J.-F.; Bogumilowicz, Y.; Holliger, P.; Rolland, G.; Billon, T. Reduced pressure-chemical vapor deposition of intrinsic and doped Ge layers on Si (001) for microelectronics and optoelectronics purposes. J. Cryst. Growth 2005, 274, 90-99.

51. Kobayashi, S.; Nishi, Y.; Saraswat, K.C. Effect of isochronal hydrogen annealing on surface roughness and threading dislocation density of epitaxial Ge films grown on Si. Thin Solid Films 2010, 518, S136-S139.

52. Lindemuth, J.R. Parallel conduction in semiconductors. III Vs Rev. 2006, 19, $28-32$.

53. Loh, T.H.; Nguyen, H.S.; Tung, C.H.; Trigg, A.D.; Lo, G.Q.; Balasubramanian, N.; Kwong, D.L.; Tripathy, S. Ultrathin low temperature SiGe buffer for the growth of high quality Ge epilayer on Si (100) by ultrahigh vacuum chemical vapor deposition. Appl. Phys. Lett. 2007, 90, doi:10.1063/1.2709993.

54. Copel, M.; Reuter, M.; Kaxiras, E.; Tromp, R. Surfactants in epitaxial growth. Phys. Rev. Lett. 1989, 63, doi:10.1103/PhysRevLett.63.632.

55. Horn-von Hoegen, M.; LeGoues, F.; Copel, M.; Reuter, M.; Tromp, R. Defect self-annihilation in surfactant-mediated epitaxial growth. Phys. Rev. Lett. 1991, 67, doi:10.1103/PhysRevLett.67.1130.

56. Tetzlaff, D.; Wietler, T.; Bugiel, E.; Osten, H. Carbon-mediated growth of thin, fully relaxed germanium films on silicon. Appl. Phys. Lett. 2012, 100, doi:10.1063/1.3675450.

57. Tetzlaff, D.; Wietler, T.; Bugiel, E.; Osten, H. Strain relaxation of thin Ge films on Si (001) grown by carbon-mediated epitaxy. J. Cryst. Growth 2013, 378, 254-258. 
58. Langdo, T.; Leitz, C.; Currie, M.; Fitzgerald, E.; Lochtefeld, A.; Antoniadis, D. High quality Ge on Si by epitaxial necking. Appl. Phys. Lett. 2000, 76, 3700-3702.

59. Bai, J.; Park, J.-S.; Cheng, Z.; Curtin, M.; Adekore, B.; Carroll, M.; Lochtefeld, A.; Dudley, M. Study of the defect elimination mechanisms in aspect ratio trapping Ge growth. Appl. Phys. Lett. 2007, 90, doi:10.1063/1.2711276.

60. Park, J.-S.; Bai, J.; Curtin, M.; Adekore, B.; Carroll, M.; Lochtefeld, A. Defect reduction of selective Ge epitaxy in trenches on $\mathrm{Si}(001)$ substrates using aspect ratio trapping. Appl. Phys. Lett. 2007, 90, doi:10.1063/1.2435603.

61. Fiorenza, J.G.; Park, J.-S.; Hydrick, J.; Li, J.; Li, J.; Curtin, M.; Carroll, M.; Lochtefeld, A. Aspect Ratio Trapping: A Unique Technology for Integrating Ge and III-Vs with Silicon CMOS. ECS Trans. 2010, 33, 963-976.

62. Niu, G. Epitaxy of Crystalline Oxides for Functional Materials Integration on Silicon; Ecole Centrale de Lyon: Ecully, France, 2010.

63. Giussani, A.; Seifarth, O.; Rodenbach, P.; Müssig, H.J.; Zaumseil, P.; Weisemöller, T.; Deiter, C.; Wollschläger, J.; Storck, P.; Schroeder, T. The influence of lattice oxygen on the initial growth behavior of heteroepitaxial Ge layers on single crystalline $\mathrm{PrO}_{2}(111) / \mathrm{Si}(111)$ support systems. J. Appl. Phys. 2008, 103, doi:10.1063/1.2870270.

64. Bojarczuk, N.A.; Copel, M.; Guha, S.; Narayanan, V.; Preisler, E.J.; Ross, F.M.; Shang, H. Epitaxial silicon and germanium on buried insulator heterostructures and devices. Appl. Phys. Lett. 2003, 83, doi:10.1063/1.1637716.

65. Ma, X.; Liu, W.; Chen, C.; Du, X.; Liu, X.; Song, Z.; Lin, C. Fabrication of high Ge content SiGe-on-insulator with low dislocation density by modified Ge condensation. Appl. Surf. Sci. 2009, 255, 7743-7748.

66. Bedell, S.; Reznicek, A.; Fogel, K.; Ott, J.; Sadana, D. Strain and lattice engineering for Ge FET devices. Mater. Sci. Semicond. Process. 2006, 9, 423-436.

67. Tezuka, T.; Sugiyama, N.; Mizuno, T.; Suzuki, M.; Takagi, S.-I. A novel fabrication technique of ultrathin and relaxed SiGe buffer layers with high Ge fraction for sub-100 nm strained silicon-on-insulator MOSFETs. Jpn. J. Appl. Phys. 2001, 40, doi:10.1143/JJAP.40.2866.

68. Liou, H.K.; Mei, P.; Gennser, U.; Yang, E.S. Effects of Ge concentration on SiGe oxidation behavior. Appl. Phys. Lett. 1991, 59, 1200-1202.

69. Chung, C.-T.; Chen, C.-W.; Lin, J.-C.; Wu, C.-C.; Chien, C.-H.; Luo, G.-L. First experimental Ge CMOS FinFETs directly on SOI substrate. In Proceedings of the Electron Devices Meeting (IEDM), 2012 IEEE International, San Francisco, CA, USA, 10-13 December 2012.

70. Dimoulas, A.; Tsipas, P. Germanium surface and interfaces (Invited Paper). Microelectron. Eng. 2009, 86, 1577-1581.

71. Caymax, M.; Houssa, M.; Pourtois, G.; Bellenger, F.; Martens, K.; Delabie, A.; Van Elshocht, S. Interface control of high-k gate dielectrics on Ge. Appl. Surf. Sci. 2008, 254, 6094-6099.

72. Xie, Q.; Deng, S.; Schaekers, M.; Lin, D.; Caymax, M.; Delabie, A.; Qu, X.-P.; Jiang, Y.-L.; Deduytsche, D.; Detavernier, C. Germanium surface passivation and atomic layer deposition of high-k dielectrics - A tutorial review on Ge-based MOS capacitors. Semicond. Sci. Technol. 2012, 27, doi:10.1088/0268-1242/27/7/074012. 
73. Choi, J.H.; Mao, Y.; Chang, J.P. Development of hafnium based high-k materials-A review. Mater. Sci. Eng. 2011, 72, 97-136.

74. Robertson, J. High dielectric constant oxides. Eur. Phys. J. Appl. Phys. 2004, 28, 265-291.

75. Wilk, G.D.; Wallace, R.M.; Anthony, J. High-k gate dielectrics: Current status and materials properties considerations. J. Appl. Phys. 2001, 89, 5243-5275.

76. Lo, S.-H.; Buchanan, D.A.; Taur, Y. Modeling and characterization of quantization, polysilicon depletion, and direct tunneling effects in MOSFETs with ultrathin oxides. IBM J. Res. Devel. 1999, 43, 327-337.

77. Lu, N.; Bai, W.; Ramirez, A.; Mouli, C.; Ritenour, A.; Lee, M.L.; Antoniadis, D.; Kwong, D.L. Ge diffusion in Ge metal oxide semiconductor with chemical vapor deposition $\mathrm{HfO}_{2}$ dielectric. Appl. Phys. Lett. 2005, 87, doi:10.1063/1.2001757.

78. Wu, N.; Zhang, Q.; Zhu, C.; Yeo, C.C.; Whang, S.J.; Chan, D.S.H.; Li, M.F.; Cho, B.J.; Chin, A.; Kwong, D.-L.; Du, A.Y.; Tung, C.H.; Balasubramanian, N. Effect of surface $\mathrm{NH}_{3}$ anneal on the physical and electrical properties of $\mathrm{HfO}_{2}$ films on Ge substrate. Appl. Phys. Lett. 2004, 84, 3741-3743.

79. Bai, W.; Lu, N.; Ritenour, A.P.; Lee, M.L.; Antoniadis, D.A.; Kwong, D.-L. The electrical properties of $\mathrm{HfO}_{2}$ dielectric on germanium and the substrate doping effect. Electron Devices IEEE Trans. 2006, 53, 2551-2558.

80. Ritenour, A.; Khakifirooz, A.; Antoniadis, D.A.; Lei, R.Z.; Tsai, W.; Dimoulas, A.; Mavrou, G.; Panayiotatos, Y. Subnanometer-equivalent-oxide-thickness germanium p-metal-oxide-semiconductor field effect transistors fabricated using molecular-beam-deposited high-k/metal gate stack. Appl. Phys. Lett. 2006, 132107:1-132107:3.

81. Seo, J.W.; Dieker, C.; Locquet, J.-P.; Mavrou, G.; Dimoulas, A. $\mathrm{HfO}_{2}$ high-k dielectrics grown on (100)Ge with ultrathin passivation layers: Structure and interfacial stability. Appl. Phys. Lett. 2005, 87, 221906:1-221906:3.

82. Delabie, A.; Puurunen, R.L.; Brijs, B.; Caymax, M.; Conard, T.; Onsia, B.; Richard, O.; Vandervorst, W.; Zhao, C.; Heyns, M.M.; et al. Atomic layer deposition of hafnium oxide on germanium substrates. J. Appl. Phys. 2005, 97, 064104:1-164104:10.

83. Profijt, H.; Potts, S.; Van de Sanden, M.; Kessels, W. Plasma-assisted atomic layer deposition: Basics, opportunities, and challenges. J. Vac. Sci. Technol. A. 2011, 29, 050801:1-050801:26.

84. Puurunen, R.L. Surface chemistry of atomic layer deposition: A case study for the trimethylaluminum/water process. J. Appl. Phys. 2005, 97, 121301:1-121301:52.

85. Martens, K.; Chi On, C.; Brammertz, G.; De Jaeger, B.; Kuzum, D.; Meuris, M.; Heyns, M.M.; Krishnamohan, T.; Saraswat, K.; Maes, H.E.; et al. On the Correct Extraction of Interface Trap Density of MOS Devices With High-Mobility Semiconductor Substrates. Electron Devices IEEE Trans. 2008, 55, 547-556.

86. Schroder, D.K. Semiconductor Material and Device Characterization, 3rd ed.; Wiley: Hoboken, NJ, USA, 2006.

87. Frank, M.M. High-k/metal gate innovations enabling continued CMOS scaling. In Proceedings of the Solid-State Device Research Conference (ESSCIRC), 2011 Proceedings of the European, Helsinki, Finland, 12-16 September 2011. 
88. Fischetti, M.V.; Neumayer, D.A.; Cartier, E.A. Effective electron mobility in Si inversion layers in metal-oxide-semiconductor systems with a high-k insulator: The role of remote phonon scattering. J. Appl. Phys. 2001, 90, 4587-4608.

89. Laikhtman, B.; Solomon, P.M. Remote phonon scattering in field-effect transistors with a high $\mathrm{k}$ insulating layer. J. Appl. Phys. 2008, 103,014501:1-014501:16.

90. Esseni, D.; Abramo, A. Modeling of electron mobility degradation by remote Coulomb scattering in ultrathin oxide MOSFETs. Electron Devices IEEE Trans. 2003, 50, 1665-1674.

91. Yamaguchi, T.; Iijima, R.; Ino, T.; Nishiyama, A.; Satake, H.; Fukushima, N. Additional scattering effects for mobility degradation in Hf-silicate gate MISFETs. In Proceedings of the Electron Devices Meeting, 2002. IEDM'02. International, San Francisco, CA, USA, 8-11 December 2002.

92. Ando, T.; Copel, M.; Bruley, J.; Frank, M.M.; Watanabe, H.; Narayanan, V. Physical origins of mobility degradation in extremely scaled $\mathrm{SiO} / \mathrm{HfO}$ gate stacks with $\mathrm{La}$ and $\mathrm{Al}$ induced dipoles. Appl. Phys. Lett. 2010, 96, 132904:1-132904:3.

93. Ando, T.; Frank, M.; Choi, K.; Choi, C.; Bruley, J.; Hopstaken, M.; Copel, M.; Cartier, E.; Kerber, A.; Callegari, A. understanding mobility mechanisms in extremely scaled $\mathrm{HfO}_{2}(\mathrm{EOT}$ $0.42 \mathrm{~nm}$ ) using remote interfacial layer scavenging technique and Vt-tuning dipoles with gate-first process. In Proceedings of the Electron Devices Meeting (IEDM), 2009 IEEE International, Baltimore, MD, USA, 7-9 December 2009.

94. Amy, F.; Beverina, A.; Chabal, Y.; Frank, M.; Shang, H.; Rivillon, S.; Hsueh, C.; Paruchuri, V.; Mo, R.; Copel, M. High-k Gate Dielectrics on Silicon and Germanium: Impact of Surface Preparation. Solid State Phenom. 2005, 103, 3-6.

95. Chi On, C.; Hyoungsub, K.; Chi, D.; McIntyre, P.C.; Saraswat, K.C. Nanoscale germanium MOS Dielectrics-part II: High-k gate dielectrics. Electron Devices IEEE Trans. 2006, 53, 1509-1516.

96. Robertson, J. Band offsets of wide-band-gap oxides and implications for future electronic devices. J. Vac. Sci. Technol. B 2000, 18, 1785-1791.

97. Robertson, J. Band offsets, Schottky barrier heights, and their effects on electronic devices. J. Vac. Sci. Technol. A 2013, 31, 050821:1-050821:18.

98. Chui, C.O.; Kim, H.; Chi, D.; Triplett, B.B.; McIntyre, P.C.; Saraswat, K.C. A sub-400/spl deg/C germanium MOSFET technology with high-/spl kappa/dielectric and metal gate, Electron Devices Meeting, 2002. IEDM'02. International, San Francisco, CA, USA, 8-11 December 2002.

99. Zhu, W.; Tamagawa, T.; Gibson, M.; Furukawa, T.; Ma, T. Effect of Al inclusion in $\mathrm{HfO}_{2}$ on the physical and electrical properties of the dielectrics. Electron Device Lett. IEEE 2002, 23, $649-651$.

100. Nishimura, T.; Lee, C.H.; Tabata, T.; Wang, S.K.; Nagashio, K.; Kita, K.; Toriumi, A. High-Electron-Mobility Ge n-Channel Metal-Oxide-Semiconductor Field-Effect Transistors with High-Pressure Oxidized $\mathrm{Y}_{2} \mathrm{O}_{3}$. Appl. Phys. Expr. 2011, 4, doi:10.1143/APEX.4.064201.

101. Mavrou, G.; Galata, S.; Tsipas, P.; Sotiropoulos, A.; Panayiotatos, Y.; Dimoulas, A.; Evangelou, E.; Seo, J.W.; Dieker, C. Electrical properties of $\mathrm{LaO}$ and $\mathrm{HfO} / \mathrm{LaO}$ gate dielectrics for germanium metal-oxide-semiconductor devices. J. Appl. Phys. 2008, 103, doi:10.1063/1.2827499. 
102. Delabie, A.; Bellenger, F.; Houssa, M.; Conard, T.; Van Elshocht, S.; Caymax, M.; Heyns, M.; Meuris, M. Effective electrical passivation of $\mathrm{Ge}(100)$ for high-k gate dielectric layers using germanium oxide. Appl. Phys. Lett. 2007, 91, 082904:1-082904:3.

103. Toriumi, A.; Tabata, T.; Hyun Lee, C.; Nishimura, T.; Kita, K.; Nagashio, K. Opportunities and challenges for Ge CMOS-Control of interfacing field on $\mathrm{Ge}$ is a key (Invited Paper). Microelectron. Eng. 2009, 86, 1571-1576.

104. Toriumi, A.; Lee, C.-H.; Nishimura, T.; Wang, S.; Kita, K.; Nagashio, K. Recent progress of Ge technology for a post-Si CMOS. ECS Trans. 2011, 35, 443-456.

105. Leys, F.E.; Bonzom, R.; Kaczer, B.; Janssens, T.; Vandervorst, W.; De Jaeger, B.; Van Steenbergen, J.; Martens, K.; Hellin, D.; Rip, J.; et al. Thin epitaxial Si films as a passivation method for $\mathrm{Ge}(100)$ : Influence of deposition temperature on Ge surface segregation and the high-k/Ge interface quality. Mater. Sci. Semicond. Process. 2006, 9, 679-684.

106. Bai, W.; Lu, N.; Kwong, D.-L. Si interlayer passivation on germanium MOS capacitors with high-k dielectric and metal gate. IEEE Electron Device Lett. 2005, 26, 378-380.

107. Wu, N.; Zhang, Q.; Zhu, C.; Chan, D.S.H.; Li, M.F.; Balasubramanian, N.; Chin, A.; Kwong, D.-L. Alternative surface passivation on germanium for metal-oxide-semiconductor applications with high-k gate dielectric. Appl. Phys. Lett. 2004, 85, doi:10.1063/1.1812835.

108. De Jaeger, B.; Bonzom, R.; Leys, F.; Richard, O.; Steenbergen, J.V.; Winderickx, G.; Moorhem, E.V.; Raskin, G.; Letertre, F.; Billon, T.; et al. Optimisation of a thin epitaxial Si layer as Ge passivation layer to demonstrate deep sub-micron n- and p-FETs on Ge-On-Insulator substrates. Microelectron. Eng. 2005, 80, 26-29.

109. Xie, R.; Zhu, C. Effects of sulfur passivation on germanium MOS capacitors with HfON gate dielectric. IEEE Electron Device Lett. 2007, 28, 976-979.

110. Ali, A.; Madan, H.; Koveshnikov, S.; Oktyabrsky, S.; Kambhampati, R.; Heeg, T.; Schlom, D.; Datta, S. Small-signal response of inversion layers in high-mobility $\operatorname{In}_{0.53} \mathrm{Ga}_{0.47} \mathrm{As}$ MOSFETs made with thin high-k dielectrics. IEEE Trans. Electron Devices 2010, 57, 742-748.

111. Engel-Herbert, R.; Hwang, Y.; Stemmer, S. Comparison of methods to quantify interface trap densities at dielectric/III-V semiconductor interfaces. J. Appl. Phys. 2010, 108, 124101:1-124101:15.

112. Nicollian, E.; Brews, J. Metal Oxide Semiconductor (MOS) Physics and Technology; John Willey \& Sons: New York, NY, USA, 1982.

113. Sun, X.; Ma, T. Electrical Characterization of Gate Traps in FETs with Ge and III-V Channels. IEEE Trans Device Mater. Reliab. 2013, 13, 463-479.

114. Prabhakaran, K.; Ogino, T. Oxidation of Ge (100) and Ge (111) surfaces: An UPS and XPS study. Surf. Sci. 1995, 325, 263-271.

115. Prabhakaran, K.; Maeda, F.; Watanabe, Y.; Ogino, T. Thermal decomposition pathway of Ge and Si oxides: observation of a distinct difference. Thin Solid Film. 2000, 369, 289-292.

116. Lee, S.; Zhu, C.; Kwong, D. Interface Engineering for High-k Ge MOSFETs. In Advanced Gate Stacks for High-Mobility Semiconductors; Springer: Berlin, Germany, 2007.

117. Dimoulas, A.; Mavrou, G.; Vellianitis, G.; Evangelou, E.; Boukos, N.; Houssa, M.; Caymax, M. $\mathrm{HfO}_{2}$ high-k gate dielectrics on $\mathrm{Ge}(100)$ by atomic oxygen beam deposition. Appl. Phys. Lett.

2005, 86, 032908:1-032908:3. 
118. Afanas'ev, V.V.; Fedorenko, Y.G.; Stesmans, A. Interface traps and dangling-bond defects in (100)Ge/HfO 2 . Appl. Phys. Lett. 2005, 87, 032107:1-032107:3.

119. Bellenger, F.; Houssa, M.; Delabie, A.; Afanasiev, V.; Conard, T.; Caymax, M.; Meuris, M.; De Meyer, K.; Heyns, M.M. Passivation of Ge ( 100 )/GeO2/high-k Gate Stacks Using Thermal Oxide Treatments. J. Electrochem. Soc. 2008, 155, G33-G38.

120. Bethge, O.; Abermann, S.; Henkel, C.; Straif, C.J.; Hutter, H.; Smoliner, J.; Bertagnolli, E. Process temperature dependent high frequency capacitance-voltage response of $\mathrm{ZrO}_{2} / \mathrm{GeO}_{2}$ /germanium capacitors. Appl. Phys. Lett. 2010, 96, 052902:1-052902:3.

121. Gu, J.; Liu, Y.; Xu, M.; Celler, G.; Gordon, R.; Ye, P. High performance atomic-layer-deposited LaLuO/Ge-on-insulator $p$-channel metal-oxide-semiconductor field-effect transistor with thermally grown $\mathrm{GeO}$ as interfacial passivation layer. Appl. Phys. Lett. 2010, 97, 012106:1-012106:3.

122. Hosoi, T.; Kutsuki, K.; Okamoto, G.; Saito, M.; Shimura, T.; Watanabe, H. Origin of flatband voltage shift and unusual minority carrier generation in thermally grown $\mathrm{GeO} / \mathrm{Ge}$ metal-oxide-semiconductor devices. Appl. Phys. Lett. 2009, 94, doi:10.1063/1.3143627.

123. Matsubara, H.; Sasada, T.; Takenaka, M.; Takagi, S. Evidence of low interface trap density in $\mathrm{GeO} / \mathrm{Ge}$ metal-oxide-semiconductor structures fabricated by thermal oxidation. Appl. Phys. Lett. 2008, 93, doi:10.1063/1.2959731.

124. Nakakita, Y.; Nakakne, R.; Sasada, T.; Takenaka, M.; Takagi, S. Interface-Controlled Self-Align Source/Drain Ge p-Channel Metal-Oxide-Semiconductor Field-Effect Transistors Fabricated Using Thermally Oxidized $\mathrm{GeO}_{2}$ Interfacial Layers. Jpn. J. Appl. Phys. 2011, 50, doi:10.1143/JJAP.50.010109.

125. Sasada, T.; Nakakita, Y.; Takenaka, M.; Takagi, S. Surface orientation dependence of interface properties of $\mathrm{GeO}_{2} / \mathrm{Ge}$ metal-oxide-semiconductor structures fabricated by thermal oxidation. J. Appl. Phys. 2009, 106, doi:10.1063/1.3234395.

126. Takagi, S.; Maeda, T.; Taoka, N.; Nishizawa, M.; Morita, Y.; Ikeda, K.; Yamashita, Y.; Nishikawa, M.; Kumagai, H.; Nakane, R.; et al. Gate dielectric formation and MIS interface characterization on Ge. Microelectron. Eng. 2007, 84, 2314-2319.

127. Xie, R.; He, W.; Yu, M.; Zhu, C. Effects of fluorine incorporation and forming gas annealing on high-k gated germanium metal-oxide-semiconductor with $\mathrm{GeO}_{2}$ surface passivation. Appl. Phys. Lett. 2008, 93, doi:10.1063/1.2966367.

128. Lin, C.-M.; Chang, H.-C.; Wong, I.-H.; Luo, S.-J.; Liu, C.W.; Hu, C. Interfacial layer reduction and high permittivity tetragonal $\mathrm{ZrO}_{2}$ on germanium reaching ultrathin $0.39 \mathrm{~nm}$ equivalent oxide thickness. Appl. Phys. Lett. 2013, 102, doi:10.1063/1.4810934.

129. Chen, Y.-T.; Lan, H.-S.; Hsu, W.; Fu, Y.-C.; Lin, J.-Y.; Liu, C.W. Strain response of high mobility germanium n-channel metal-oxide-semiconductor field-effect transistors on (001) substrates. Appl. Phys. Lett. 2011, 99, doi:10.1063/1.3604417.

130. Lee, C.; Nishimura, T.; Tabata, T.; Wang, S.; Nagashio, K.; Kita, K.; Toriumi, A. Ge MOSFETs performance: Impact of Ge interface passivation. In Proceedings of the Electron Devices Meeting (IEDM), 2010 IEEE International, San Francisco, CA, USA, 6-8 December 2010.

131. Baldovino, S.; Molle, A.; Fanciulli, M. Influence of the oxidizing species on the Ge dangling bonds at the (100)Ge/GeO $\mathrm{GeO}_{2}$ interface. Appl. Phys. Lett. 2010, 96, 222110:1-222110:3. 
132. Delabie, A.; Alian, A.; Bellenger, F.; Caymax, M.; Conard, T.; Franquet, A.; Sioncke, S.; Van Elshocht, S.; Heyns, M.; Meuris, M. $\mathrm{H}_{2} \mathrm{O}$-and $\mathrm{O}_{3}$-Based Atomic Layer Deposition of High-k Dielectric Films on $\mathrm{GeO}_{2}$ Passivation Layers. J. Electrochem. Soc. 2009, 156, G163-G167.

133. Kuzum, D.; Krishnamohan, T.; Pethe, A.J.; Okyay, A.K.; Oshima, Y.; Sun, Y.; McVittie, J.P.; Pianetta, P.A.; McIntyre, P.C.; Saraswat, K.C. Ge-interface engineering with ozone oxidation for low interface-state density. IEEE Electron Device Lett. 2008, 29, 328-330.

134. Kuzum, D.; Park, J.-H.; Krishnamohan, T.; Wong, H.-S.; Saraswat, K.C. The effect of donor/acceptor nature of interface traps on Ge MOSFET characteristics. IEEE Trans. Electron Devices 2011, 58, 1015-1022.

135. Molle, A.; Bhuiyan, M.N.K.; Tallarida, G.; Fanciulli, M. In situ chemical and structural investigations of the oxidation of $\mathrm{Ge}(001)$ substrates by atomic oxygen. Appl. Phys. Lett. 2006, 89, 083504:1-083504:3.

136. Tsipas, P.; Volkos, S.N.; Sotiropoulos, A.; Galata, S.F.; Mavrou, G.; Tsoutsou, D.; Panayiotatos, Y.; Dimoulas, A.; Marchiori, C.; Fompeyrine, J. Germanium-induced stabilization of a very high-k zirconia phase in $\mathrm{ZrO}_{2} / \mathrm{GeO}_{2}$ gate stacks. Appl. Phys. Lett. 2008, 93, doi:10.1063/1.2977555.

137. Lee, C.H.; Tabata, T.; Nishimura, T.; Nagashio, K.; Kita, K.; Toriumi, A. Ge/GeO $\mathrm{G}_{2}$ Interface Control with High Pressure Oxidation for Improving Electrical Characteristics. ECS Trans. 2009, 19, 165-173.

138. Kobayashi, M.; Thareja, G.; Ishibashi, M.; Sun, Y.; Griffin, P.; McVittie, J.; Pianetta, P.; Saraswat, K.; Nishi, Y. Radical oxidation of germanium for interface gate dielectric $\mathrm{GeO}_{2}$ formation in metal-insulator-semiconductor gate stack. J. Appl. Phys. 2009, 106, doi:10.1063/1.3259047.

139. Fukuda, Y.; Yazaki, Y.; Otani, Y.; Sato, T.; Toyota, H.; Ono, T. Low-Temperature Formation of High-Quality $\mathrm{GeO}_{2}$ Interlayer for High-k Gate Dielectrics/Ge by Electron-Cyclotron-Resonance Plasma Techniques. IEEE Trans. Electron Devices 2010, 57, 282-287.

140. Van Elshocht, S.; Brijs, B.; Caymax, M.; Conard, T.; Chiarella, T.; De Gendt, S.; De Jaeger, B.; Kubicek, S.; Meuris, M.; Onsia, B.; et al. Deposition of $\mathrm{HfO}_{2}$ on germanium and the impact of surface pretreatments. Appl. Phys. Lett. 2004, 85, 3824-3826.

141. Swaminathan, S.; Shandalov, M.; Oshima, Y.; McIntyre, P.C. Bilayer metal oxide gate insulators for scaled Ge-channel metal-oxide-semiconductor devices. Appl. Phys. Lett. 2010, 96, 082904:1-082904:3.

142. Leys, F.E.; Bonzom, R.; Loo, R.; Richard, O.; De Jaeger, B.; Van Steenbergen, J.; Dessein, K.; Conard, T.; Rip, J.; Bender, H.; et al. Epitaxy solutions for Ge MOS technology. Thin Solid Film 2006, 508, 292-296.

143. Dimoulas, A.; Tsipas, P.; Sotiropoulos, A.; Evangelou, E.K. Fermi-level pinning and charge neutrality level in germanium. Appl. Phys. Lett. 2006, 89, doi:10.1063/1.2410241.

144. Nishimura, T.; Kita, K.; Toriumi, A. Evidence for strong Fermi-level pinning due to metal-induced gap states at metal/germanium interface. Appl. Phys. Lett. 2007, 91, doi:10.1063/1.2789701.

145. Lin, J.Y.J.; Roy, A.M.; Nainani, A.; Sun, Y.; Saraswat, K.C. Increase in current density for metal contacts to n-germanium by inserting $\mathrm{TiO}_{2}$ interfacial layer to reduce Schottky barrier height. Appl. Phys. Lett. 2011, 98, doi:10.1063/1.3562305. 
146. Zhou, Y.; Ogawa, M.; Han, X.; Wang, K.L. Alleviation of Fermi-level pinning effect on metal/germanium interface by insertion of an ultrathin aluminum oxide. Appl. Phys. Lett. 2008, 93, doi:10.1063/1.3028343.

147. Kobayashi, M.; Kinoshita, A.; Saraswat, K.; Wong, H.-S.P.; Nishi, Y. Fermi level depinning in metal/Ge Schottky junction for metal source/drain $\mathrm{Ge}$ metal-oxide-semiconductor field-effect-transistor application. J. Appl. Phys. 2009, 105, doi:10.1063/1.3065990.

148. Jain, N.; Zhu, Y.; Maurya, D.; Varghese, R.; Priya, S.; Hudait, M. Interfacial band alignment and structural properties of nanoscale $\mathrm{TiO}_{2}$ thin films for integration with epitaxial crystallographic oriented germanium. J. Appl. Phys. 2014, 115, 024303, doi:10.1063/1.4861137.

149. Lieten, R.R.; Degroote, S.; Kuijk, M.; Borghs, G. Ohmic contact formation on n-type Ge. Appl. Phys. Lett. 2008, 92, 022106:1-022106:3.

150. Takahashi, T.; Nishimura, T.; Chen, L.; Sakata, S.; Kita, K.; Toriumi, A. Proof of Ge-interfacing concepts for metal/high-k/Ge CMOS-Ge-intimate material selection and interface conscious process flow. In Proceedings of the Electron Devices Meeting, 2007. IEDM 2007. IEEE International, Washington, DC, USA, 10-12 December 2007.

151. Nishimura, T.; Kita, K.; Toriumi, A. A significant shift of Schottky barrier heights at strongly pinned metal/germanium interface by inserting an ultra-thin insulating film. Appl. Phys. Expr. 2008, 1, doi:10.1143/APEX.1.051406.

152. Lee, D.; Raghunathan, S.; Wilson, R.J.; Nikonov, D.E.; Saraswat, K.; Wang, S.X. The influence of Fermi level pinning/depinning on the Schottky barrier height and contact resistance in $\mathrm{Ge} / \mathrm{CoFeB}$ and $\mathrm{Ge} / \mathrm{MgO} / \mathrm{CoFeB}$ structures. Appl. Phys. Lett. 2010, 96, 052514:1-052514:13.

153. Zhou, Y.; Han, W.; Wang, Y.; Xiu, F.; Zou, J.; Kawakami, R.; Wang, K. Investigating the origin of Fermi level pinning in Ge Schottky junctions using epitaxially grown ultrathin $\mathrm{MgO}$ films. Appl. Phys. Lett. 2010, 96, doi:10.1063/1.3357423.

154. Li, Z.; An, X.; Yun, Q.; Lin, M.; Zhang, X.; Huang, R. Tuning Schottky Barrier Height in Metal/n-Type Germanium by Inserting an Ultrathin Yttrium Oxide Film. ECS Solid State Lett. 2012, 1, Q33-Q34.

155. Hudait, M.K. (Invited) Heterogeneously Integrated III-V on Silicon for Future Nanoelectronics. ECS Trans. 2012, 45, 581-594.

156. Datta, S.; Dewey, G.; Fastenau, J.M.; Hudait, M.K.; Loubychev, D.; Liu, W.K.; Radosavljevic, M.; Rachmady, W.; Chau, R. Ultrahigh-Speed 0.5 V Supply Voltage $\operatorname{In}_{0.7} \mathrm{Ga}_{0.3}$ As Quantum-Well Transistors on Silicon Substrate. IEEE Electron Device Lett. 2007, 28, 685-687.

157. Radosavljevic, M.; Ashley, T.; Andreev, A.; Coomber, S.D.; Dewey, G.; Emeny, M.T.; Fearn, M.; Hayes, D.G.; Hilton, K.P.; Hudait, M.K.; et al. High-performance $40 \mathrm{~nm}$ gate length InSb $p$-channel compressively strained quantum well field effect transistors for low-power $(V C C=0.5 \mathrm{~V})$ logic applications. In Proceedings of the Electron Devices Meeting, 2008. IEDM 2008. IEEE International 2008, San Francisco, CA, USA, 15-17 December 2008.

158. Edirisooriya, M.; Mishima, T.D.; Gaspe, C.K.; Bottoms, K.; Hauenstein, R.J.; Santos, M.B. InSb quantum-well structures for electronic device applications. J. Cryst. Growth 2009, 311, 1972-1975.

159. Klem, J.F.; Lott, J.A.; Schirber, J.E.; Kurtz, S.R.; Lin, S.Y. Strained quantum-well modulation-doped InGaSb/AlGaSb structures grown my molecular beam epitaxy. J. Electron. Mater. 1993, 22, 315-321. 
160. Boos, J.B.; Bennett, B.R.; Papanicolaou, N.A.; Ancona, M.G.; Champlain, J.G.; Chou, Y.C.; Lange, M.D.; Yang, J.M.; Bass, R.; Park, D.; et al. Sb-based $n$ - and $p$-channel Heterostructure FETs for High-Speed, Low-Power Applications. IEICE Trans. Electron. 2008, E91-C, 1050-1057.

161. Klem, J.F.; Lovejoy, M.L. AlGaAsSb/InGaSb quantum-well heterostructures for p-channel field-effect transistors. J. Vac. Sci. Technol. B 1995, 13, 702-705.

162. Lott, J.A.; Klem, J.F.; Wendt, J.R. Strained $p$-channel InGaSb/AlGaSb modulation-doped field-effect transistors. Electron. Lett. 1992, 28, 1459-1460.

163. Liao, C.; Cheng, K.Y. Hole mobility in pseudomorphic InGaSb quantum well modulation doped with carbon. J. Vac. Sci. Technol. B 2010, 28, C3C29-C3C32.

164. Nainani, A.; Raghunathan, S.; Witte, D.; Kobayashi, M.; Irisawa, T.; Krishnamohan, T.; Saraswat, K.; Bennett, B.R.; Ancona, M.G.; Boos, J.B. Engineering of strained III-V heterostructures for high hole mobility. In Proceedings of the Electron Devices Meeting (IEDM) 2009 IEEE International, Baltimore, MD, USA, 7-9 December 2009.

165. Bennett, B.R.; Ancona, M.G.; Boos, J.B. Compound Semiconductors for Low-Power $p$-Channel Field-Effect Transistors. MRS Bull. 2009, 34, 530-536.

166. Bennett, B.R.; Ancona, M.G.; Boos, J.B.; Shanabrook, B.V. Mobility enhancement in strained p-InGaSb quantum wells. Appl. Phys. Lett. 2007, 91, 042104:1-042104:3.

167. Boos, J.B.; Bennett, B.R.; Papanicolaou, N.A.; Ancona, M.G.; Champlain, J.G.; Bass, R.; Shanabrook, B.V. High mobility $p$-channel HFETs using strained Sb-based materials. Electron. Lett. 2007, 43, 834-835.

168. Bennett, B.R.; Ancona, M.G.; Boos, J.B.; Canedy, C.B.; Khan, S.A. Strained GaSb/AlAsSb quantum wells for $p$-channel field-effect transistors. J. Cryst. Growth 2008, 311, 47-53.

169. Yoh, K.; Kiyomi, K.; Taniguchi, H.; Yaho, M.; Ihoue, M. In a p-channel GaSb heterojunction field-effect transistor based on a vertically integrated complementary circuit structure. Gallium Arsenide and Related Compounds 1991, Proceedings of the Eighteenth INT Symposium, Seattle, WA, USA, 9-12 September 1991.

170. Luo, L.F.; Longenbach, K.F.; Wang, W.I. p-Channel modulation-doped GaSb field-effect transistors. Electron. Lett. 1991, 27, 472-474.

171. Yoh, K.; Taniguchi, H.; Kiyomi, K.; Inoue, M. Complementary InAs n-channel and GaSb p-channel quantum well heterojunction field-effect transistors. Jpn J. Appl. Phys. 1991, 30, 3833-3836.

172. Yoh, K.; Kiyomi, K.; Yano, M.; Inoue, M. Molecular beam epitaxial groan InAs/GaSb double quantum-wells for complementary heterojunction field-effect transistors. J. Cryst. Growth 1993, 127, 29-35.

173. Nainani, A.; Krishnamohan, T.; Kim, D.; Saraswat, K. In hole mobility and its enhancement with strain for technologically relevant III-V semiconductors. International Conference on Simulation of Semiconductor Processes and Devices (SISPAD), San Diego, CA, USA, 9-11 September 2009.

174. Jaffe, M.; Sekiguchi, Y.; Singh, J. Theoretial formalism to understand the role of strain in the tailoring of hole masses in p-type $\operatorname{In}_{x} \mathrm{Ga}_{1-x} \mathrm{As}$ (on GaAs substrates) and $\operatorname{In}_{0.53+x} \mathrm{Ga}_{0.47-x} \mathrm{As}$ (on InP substrates) modulation-doped field-effect transistors. Appl. Phys. Lett. 1987, 51, 1943-1945.

175. Hinckley, J.M.; Singh, J. Theoretical investigation of hole transport in strained III-V semiconductors- Application to GaAs. Appl. Phys. Lett. 1988, 53, 785-787. 
176. Jaffe, M.; Oh, J.E.; Pamulapati, J.; Singh, J.; Bhattacharya, P. In-plane hole effective masses in $\mathrm{In}_{x} \mathrm{Ga}_{1-x} \mathrm{As} / \mathrm{Al}_{0.15} \mathrm{Ga}_{0.85} \mathrm{As}$ modulation-doped heterostructures. Appl. Phys. Lett. 1989, 54, 2345-2347.

177. Jaffe, M.; Singh, J. Band-structure and charge control studies of n-type and p-type pseudomorphic modulation-doped field-effect transistors. J. Appl. Phys. 1989, 65, 329-338.

178. Singh, J. The tailoring of impact ionization phenomenon using pseudomorphic structures-applications to InGaAlAs on GaAs and InP substrates. Semicond. Sci. Technol. 1992, 7, B509-B511.

179. Fischetti, M.V.; Laux, S.E. Band structure, deformation potentials, and carrier mobility in strained Si, Ge, and SiGe alloys. J. Appl. Phys. 1996, 80, 2234-2252.

180. Kim, J.; Fischetti, M.V. Electronic band structure calculations for biaxially straines Si, Ge and III-V semiconductors. J. Appl. Phys. 2010, 108, 013710:1-013711:5.

181. Zhang, Y.; Fischetti, M.V. Calculation of hole mobility in Ge and III-V p-channels. In Proceedings of the 13th International Workshop on Computational Electronics (IWCE), Beijing, China, 27-29 May 2009.

182. Datta, S.; Ashley, T.; Brask, J.; Buckle, L.; Doczy, M.; Emeny, M.; Hayes, D.; Hilton, K.; Jefferies, R.; Martin, R.; et al. $85 \mathrm{~nm}$ gate length enhancement and depletion mode InSb quantum well transistors for ultra high speed and very low power digital logic applications. In Proceedings of the IEEE International Electron Devices Meeting, Washington, DC, USA, 5 December 2005.

183. Trinh, H.D.; Chang, E.Y.; Wu, P.W.; Wong, Y.Y.; Chang, C.T.; Hsieh, Y.F.; Yu, C.C.; Nguyen, H.Q.; Lin, Y.C.; Lin, K.L.; et al. The influences of surface treatment and gas annealing conditions on the inversion behaviors of the atomic-layer-deposition $\mathrm{Al}_{2} \mathrm{O}_{3} / \mathrm{n}-\mathrm{In}_{0.53} \mathrm{Ga}_{0.47} \mathrm{As}$ metal-oxide-semiconductor capacitor. Appl. Phys. Lett. 2010, 97, doi:10.1063/1.3467813.

184. Ye, P.D. Main determinants for III-V metal-oxide-semiconductor field-effect transistors (invited). J. Vac. Sci. Technol. A 2008, 26, 697-704.

185. Ali, A.; Madan, H.S.; Kirk, A.P.; Zhao, D.A.; Mourey, D.A.; Hudait, M.K.; Wallace, R.M.; Jackson, T.N.; Bennett, B.R.; Boos, J.B.; et al. Fermi level unpinning of GaSb (100) using plasma enhanced atomic layer deposition of $\mathrm{Al}_{2} \mathrm{O}_{3}$. Appl. Phys. Lett. 2010, 97, doi:10.1063/1.3492847.

186. Hudait, M.K.; Lin, Y.; Palmisiano, M.N.; Tivarus, C.; Pelz, J.P.; Ringel, S.A. Comparison of mixed anion, InAsyP1-y and mixed cation, $\mathrm{In}_{x} \mathrm{Al}_{1-x} \mathrm{As}$ metamorphic buffers grown by molecular beam epitaxy on (100)InP substrates. J. Appl. Phys. 2004, 95, 3952-3960.

187. Baraskar, A.K.; Wistey, M.A.; Jain, V.; Singisetti, U.; Burek, G.; Thibeault, B.J.; Lee, Y.J.; Gossard, A.C.; Rodwell, M.J.W. Ultralow resistance, nonalloyed Ohmic contacts to n-InGaAs. J. Vac. Sci. Technol. B 2009, 27, 2036-2039.

188. Singisetti, U.; Wistey, M.A.; Burek, G.J.; Baraskar, A.K.; Thibeault, B.J.; Gossard, A.C.; Rodwell, M.J.W.; Shin, B.; Kim, E.J.; McIntyre, P.C.; et al. $\operatorname{In}_{0.53} \mathrm{Ga}_{0.47}$ As Channel MOSFETs with Self-Aligned InAs Source/Drain Formed by MEE Regrowth. IEEE Electron Device Lett. 2009, 30, 1128-1130.

189. Kudo, M.; Matsumoto, H.; Tanimoto, T.; Mishima, T.; Ohbu, I. Improved hole transport properties of highly strained $\mathrm{In}_{0.35} \mathrm{Ga}_{0.65}$ As channel double-modulation-doped structures grown by MBE on GaAs. J. Cryst. Growth 1997, 175-176, 910-914. 
190. Kudo, M.; Mishima, T.; Matsumoto, H.; Ohbu, I.; Tanimoto, T. Highly strained $\mathrm{In}_{0.35} \mathrm{Ga}_{0.65} \mathrm{As} / \mathrm{GaAs}$ layers grown by molecular beam epitaxy for high hole mobility transistors. $J$. Electron. Mater. 1996, 25, 944-947.

191. Nagaiah, P.; Tokranov, V.; Yakimov, M.; Oktyabrsky, S. Strained quantum wells for p-channel InGaAs CMOS. MRS Proceedings, San Francisco, CA, USA, 24-28 March 2008.

192. Morris, R.J.H.; Grasby, T.J.; Hammond, R.; Myronov, M.; Mironov, O.A.; Leadley, D.R.; Whall, T.E.; Parker, E.H.C.; Currie, M.T.; Leitz, C.W.; et al. High conductance Ge p-channel heterostructures realized by hybrid epitaxial growth. Semicond. Sci. Technol. 2004, 19, L106-L109.

193. Myronov, M.; Sawano, K.; Shiraki, Y.; Mouri, T.; Itoh, K.M. Observation of two-dimensional hole gas with mobility and carrier density exceeding those of two-dimensional electron gas at room temperature in the SiGe heterostructures. Appl. Phys. Lett. 2007, 91, 082108:1-082108:3.

194. Myronov, M.; Leadley, D.R.; Shiraki, Y. High mobility holes in a strained Ge quantum well grown on a thin and relaxed $\mathrm{Si}_{0.4} \mathrm{Ge}_{0.6} / \mathrm{LT}-\mathrm{Si}_{0.4} \mathrm{Ge}_{0.6} / \mathrm{Si}(001)$ virtual substrate. Appl. Phys. Lett. 2009, 94, doi:10.1063/1.3090034.

195. Myronov, M.; Irisawa, T.; Mironov, O.A.; Koh, S.; Shiraki, Y.; Whall, T.E.; Parker, E.H.C. Extremely high room-temperature two-dimensional hole gas mobility in $\mathrm{Ge} / \mathrm{Si}_{0.33} \mathrm{Ge}_{0.67} /$ Si(001) p-type modulation-doped heterostructures. Appl. Phys. Lett. 2002, 80, 3117-3119.

196. Irisawa, T.; Tokumitsu, S.; Hattori, T.; Nakagawa, K.; Koh, S.; Shiraki, Y. Ultrahigh room-temperature hole Hall and effective mobility in $\mathrm{Si}_{0.3} \mathrm{Ge}_{0.7} / \mathrm{Ge}_{\mathrm{Si}} / \mathrm{Si}_{0.3} \mathrm{Ge}_{0.7}$ heterostructures. Appl. Phys. Lett. 2002, 81, 847-849.

197. Myronov, M.; Sawano, K.; Shiraki, Y. Enhancement of hole mobility and carrier density in Ge quantum well of SiGe heterostructure via implementation of double-side modulation doping. Appl. Phys. Lett. 2006, 88, doi:10.1063/1.2215633.

198. Bennett, B.R.; Magno, R.; Boos, J.B.; Kruppa, W.; Ancona, M.G. Antimonide-based compound semiconductors for electronic devices: A review. Solid State Electron. 2005, 49, 1875-1895.

199. Chau, R.; Datta, S.; Doczy, M.; Doyle, B.; Jin, B.; Kavalieros, J.; Majumdar, A.; Metz, M.; Radosavljevic, M. Benchmarking Nanotechnology for High-Performance and Low-Power Logic Transistor Applications. IEEE Trans. Nanotechnol. 2005, 4, 153-158.

200. Duriez, B.; Vellianitis, G.; van Dal, M.J.H.; Doornbos, G.; Oxland, R.; Bhuwalka, K.K.; Holland, M.; Chang, Y.S.; Hsieh, C.H.; Yin, K.M.; et al. Scaled p-channel Ge FinFET with optimized gate stack and record performance integrated on $300 \mathrm{~mm}$ Si wafers. Electron Devices Meeting (IEDM), 2013 IEEE International, Washington, DC, USA, 9-11 December 2013.

(C) 2014 by the authors; licensee MDPI, Basel, Switzerland. This article is an open access article distributed under the terms and conditions of the Creative Commons Attribution license (http://creativecommons.org/licenses/by/3.0/). 